Article

\title{
Petrology of Mafic Dykes from the Njimom Area (West-Cameroon): A Contribution to the Characterization of Late Paleozoic and Mesozoic Magmatism in the Southern Continental Part of the Cameroon Volcanic Line
}

\author{
Noël-Aimée Kouamo Keutchafo ${ }^{1}$, Nicole Armelle Wambo Simeni ${ }^{2}$, Brillant Kandzi Nforba ${ }^{1}$, \\ Agathe Arrissa Noucoucouk ${ }^{3}$, Josiane Demlabin Sonmo ${ }^{1}$, Depesquidoux Tchato Tchaptchet ${ }^{1}$, \\ Jean Pierre Tchouankoue ${ }^{1, *}$ and Ciro Cucciniello ${ }^{4, *}$ D
}

\section{check for}

updates

Citation: Keutchafo, N.-A.K.;

Simeni, N.A.W.; Nforba, B.K.;

Noucoucouk, A.A.; Sonmo, J.D.; Tchaptchet, D.T.; Tchouankoue, J.P.; Cucciniello, C. Petrology of Mafic Dykes from the Njimom Area (West-Cameroon): A Contribution to the Characterization of Late Paleozoic and Mesozoic Magmatism in the Southern Continental Part of the Cameroon Volcanic Line.

Geosciences 2022, 12, 12.

https://doi.org/10.3390/ geosciences12010012

Academic Editors: Ian Smith and Jesus Martinez-Frias

Received: 8 November 2021 Accepted: 23 December 2021 Published: 28 December 2021

Publisher's Note: MDPI stays neutral with regard to jurisdictional claims in published maps and institutional affiliations.

Copyright: (c) 2021 by the authors Licensee MDPI, Basel, Switzerland. This article is an open access article distributed under the terms and conditions of the Creative Commons Attribution (CC BY) license (https:// creativecommons.org/licenses/by/ $4.0 /)$
1 Department of Earth Sciences, University of Yaoundé I, Yaounde P.O. Box 812, Cameroon; noelaimeekeutchafo@gmail.com (N.-A.K.K.); brilliantnforba@gmail.com (B.K.N.); sonmojosiane@yahoo.fr (J.D.S.); depesquidoux@yahoo.fr (D.T.T.)

2 Faculty of Science, University of Ngaoundéré, Ngaoundere P.O. Box 454, Cameroon; swannie2009@yahoo.fr 3 Centro de Tecnologia e Geosciências/UFPE, Departamento de Geologia, Av. Acadêmico Hélio Ramos s/n Cidade Universitaria, Recife 50740-530, Brazil; agathenoucoucouk@yahoo.com

4 Dipartimento di Scienze della Terra, dell'Ambiente e delle Risorse (DiSTAR), Università di Napoli Federico II, Complesso Universitario di Monte Sant'Angelo, Via Vicinale Cupa Cintia 21, 80126 Napoli, Italy

* Correspondence: tchouankoue@yahoo.com (J.P.T.); ciro.cucciniello@unina.it (C.C.)

\begin{abstract}
In the western Cameroon, crop out several dyke swarms of Paleozoic-Mesozoic age. These dykes intrude the Precambrian basement in the southern continental part of the Cretaceous Cameroon Volcanic Line. In the Njimom area, two groups of mafic dykes that crosscut the Neoproterozoic basement rocks have been observed. A first group intrudes the mylonites whereas the second group intrudes the granites. The dykes are alkaline basalts and hawaiites. The mineralogical assemblage of both groups of dykes consists of plagioclase, clinopyroxene, altered olivine, and opaque oxides. The dykes that cross-cut the Precambrian mylonitic gneisses show moderate $\mathrm{TiO}_{2}(1.7-2.0$ wt.\%), low $\mathrm{MgO}$ (4.4-7.1 wt.\%), and compatible trace element concentrations (e.g., $\mathrm{Cr}=70-180 \mathrm{ppm}$; $\mathrm{Ni}=30-110 \mathrm{ppm})$. The dykes that intrude the granites have $\mathrm{TiO}_{2}$ contents between 2.3 and 2.5 wt. $\%$ and moderate compatible trace element concentrations (e.g., $\mathrm{Cr}=260-280 \mathrm{ppm} ; \mathrm{Ni}=170-230 \mathrm{ppm}$ ). $\mathrm{MgO}$ varies from 5.9 to $9.2 \mathrm{wt} . \%$. All mafic dykes are enriched in light lanthanide element and show moderate $\mathrm{Zr} / \mathrm{Nb}$ and high $\mathrm{Zr} / \mathrm{Y}, \mathrm{Nb} / \mathrm{Yb}$, and $\mathrm{Ti} / \mathrm{V}$ ratios similar to those of average ocean island basalt (OIB)-type magmas. Some dykes that intrude the mylonites show evidence of contamination by continental crust. The composition of the clinopyroxenes of the dykes that intrude the mylonites clearly indicate different and unrelated parental magmas from dykes that intrude the granites. Contents and fractionation of the least and the most incompatible elements suggest low degrees of partial melting $(3-5 \%)$ of heterogeneous source slightly enriched in incompatible elements in the spinel stability field. The geochemical features of Njimom dykes (in particular the dykes that intrude the granites) are similar to those of Paleozoic and Mesozoic dykes recorded in the southern continental part of the Cameroon Volcanic Line, suggesting multiple reactivations of pre-existing fractures that resulted in the fragmentation of western Gondwana and the opening of the South Atlantic Ocean.
\end{abstract}

Keywords: mafic dykes; mineralogy; geochemistry; petrogenesis; Njimom; western Cameroon

\section{Introduction}

Volcanic activity in Cameroon is concentrated along the well-known Cameroon Volcanic Line (CVL), an alignment of oceanic and continental magmatic centers that straddle the boundary continental/oceanic crust in Central Africa (e.g., [1]). The CVL extends 
(with a N30 ${ }^{\circ}$ E direction) over more than $1000 \mathrm{~km}$ in Cameroon, Equatorial Guinea, Sao Tome, and Principe. According to some authors, the CVL extends also in Nigeria in the Biu Plateau and in Chad (e.g., [2,3]). Volcanic rocks of the Cameroon Line cover the Precambrian basement of Neoproterozoic age (e.g., [4]). In the southern continental part of the CVL, the Paleozoic-Mesozoic dyke swarms show mineralogical and geochemical characteristics different from the common CVL rocks (e.g., [5,6]). While in CVL the rocks are mainly alkaline, the dykes are essentially transitional and/or tholeiitic. The oldest basalts of the CVL are of Tertiary age while the dykes span over Paleozoic and Mesozoic eras with youngest know age (192.10 $\pm 7.45 \mathrm{Ma})$ being found for the Kendem dyke which is the only NW-SE dyke dated [7]. On a structural basis, orientations of basaltic dykes are aggregate to the range NNE-ENE with a predominance of the ENE orientation which is locally known as the Adamawa Shear Zone (ASZ) which runs from the Gulf of Guinea to Sudan and in a pre-drift reconstitution, is thought to prolong to Brazil as the Pernambuco Shear Zone. Tectonic studies evidence a Riedel fracture model with a dextral shearing along the ASZ (e.g., [8,9]) with a NW-SE shortening $(\sigma 1)$ associated to the formation of the Benue aulacogen, a NE-SW stretching ( $\sigma 3$ ) corresponding to the CVL. Our field works along the ASZ in the Njimom area indicate the existence of NW-SE oriented basaltic dyke swarms. Here we present a study of the Njimom dykes. With field, petrographic and whole-rock geochemical (major and trace element) data, we decipher the petrogenesis of this dyke suite and the signification of the NW-SE dykes group in the framework of the opening of the Southern Atlantic Ocean.

\section{Geological Setting}

The studied dykes are located to the West of the city of Njimom, in the southern continental part of the CVL (Figures 1 and 2). The area lies on a $\sim 0.6$ Ga granitic-gneissic basement (e.g., $[10,11])$ strongly mylonitized along the Central Cameroonian Shear Zone, a segment of the regional Adamawa Shear Zone. The rocks that compose the Pan-African basement in the area are coarse grained granitoids, granoblastic orthogneisses, migmatites, metagabbros, and mylonites [12]. Structural features and kinematic indicators point to a syntectonic emplacement of the basement rocks and provide detailed information on the relative timing of deformation $[13,14]$. Younger mafic dykes and quartz-feldspathic veins crosscut mylonites and granites while amphibolitic enclaves are widespread in gneisses. Migmatites are associated with orthogneisses and display high $\mathrm{K}$ to shoshonitic characters [12]. The spatial distinction in pre- to syn-orogenic magmatism allows recognition of a north to south potassium increasing trend, compatible with the existence of a northwestern trending Pan-African subduction (e.g., [15]). This sheared margin is marked by the superposition of two mylonitic foliations operating in opposing sense (at constant direction), under high and low metamorphic conditions (e.g., [13]). Volcanic activity in the studied area consists of relics of basaltic plateau (ca. $51 \mathrm{Ma}$; [16]) of the CVL in the Foumban area (60 $\mathrm{km}$ to the SW of the dykes studied in this work). 


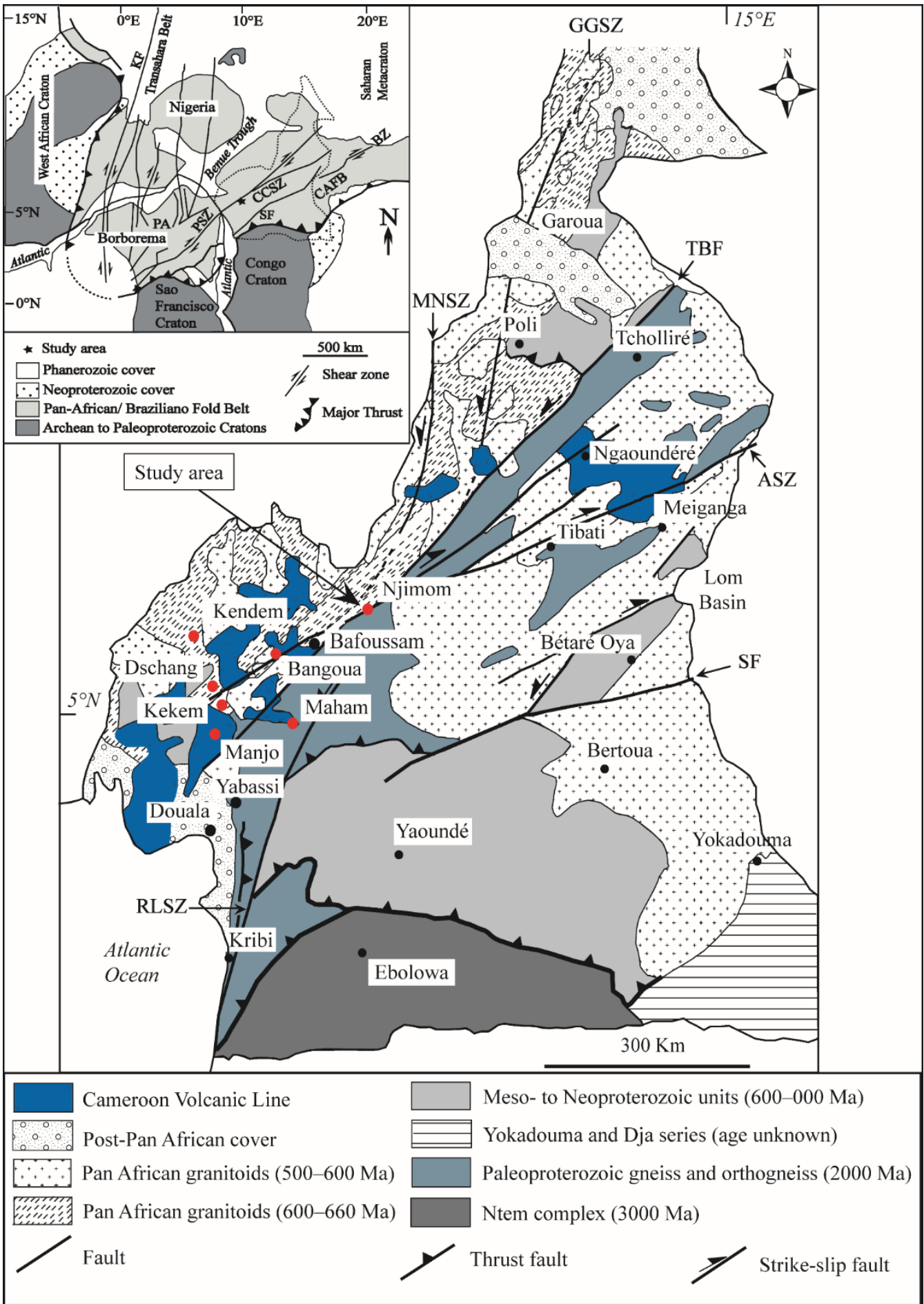

Figure 1. Geological sketch map of Cameroon [17]. CCSZ = Central Cameroon shear zone, $\mathrm{SF}=$ Sanaga Fault, $\mathrm{TBF}=$ Tcholliré-Banyo fault, $\mathrm{RLSZ}=$ Rocher du Loup shear zone, ASZ = Adamawa shear zone, GGSZ = Godé-Gormaya shear zone, MNZ = Mayo Nolti shear zone. The insert is the position of the studied area in pre-drift reconstruction [11]. PA = Patos shear zone, KF = Kandi Fault, PSZ = Pernambuco shear zone. The locations of the mafic dyke swarms (Njimom, Kendem, Dschang, Bangangte, Manjo, Nyos) are also shown. 


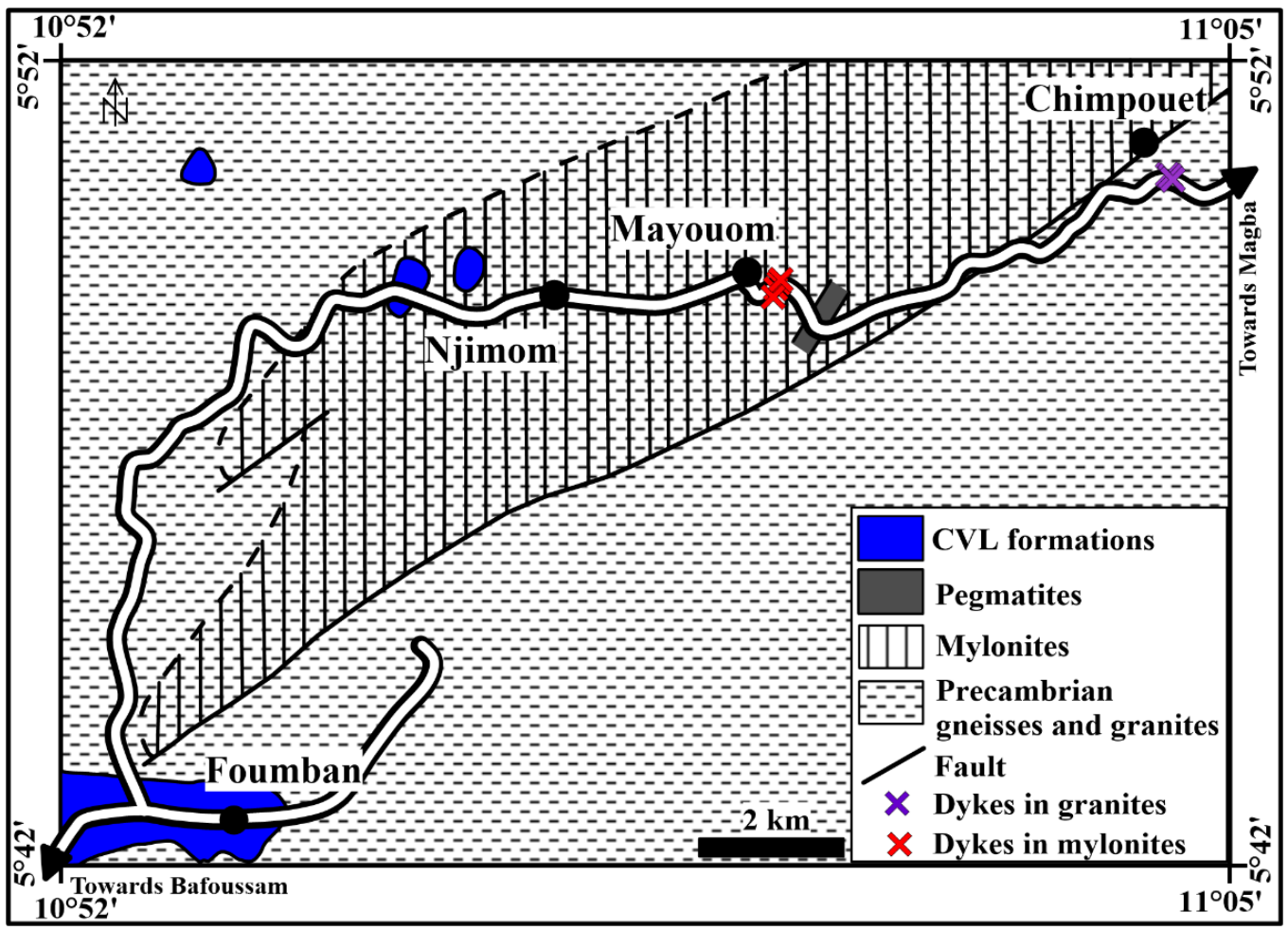

Figure 2. Geology sketch map of the Njimom area with location of the studied samples.

\section{Field Occurrence and Petrography}

The Njimom dykes strike mainly $135^{\circ} \mathrm{N}$ and $155^{\circ} \mathrm{N}$ and cross-cut respectively mylonitic gneisses and granites of the Precambrian basement of the area. The dykes are well exposed along the Foumban-Magba newly tarred road. The dykes in mylonites $\left(5^{\circ} 49^{\prime} 58.8^{\prime \prime} \mathrm{N} / 10^{\circ} 59^{\prime} 50.0^{\prime \prime} \mathrm{E}, 5^{\circ} 50^{\prime} 00.1^{\prime \prime} \mathrm{N} / 10^{\circ} 59^{\prime} 51.7^{\prime \prime} \mathrm{E}\right)$ occur as slabs and blocks (Figure 3a) with a thickness between 0.5 and $6 \mathrm{~m}$. The second group of dykes $\left(5^{\circ} 51^{\prime} 04.3^{\prime \prime} \mathrm{N} / 11^{\circ} 04^{\prime} 18.6^{\prime \prime} \mathrm{E}\right.$, $5^{\circ} 51^{\prime} 04.2^{\prime \prime} \mathrm{N} / 11^{\circ} 04^{\prime} 16.8^{\prime \prime} \mathrm{E}$ ) with a thicknesses between 0.2 to $1 \mathrm{~m}$ cuts sub-parallel to parallel (to the NW-SE direction) the granites (Figure 3b).
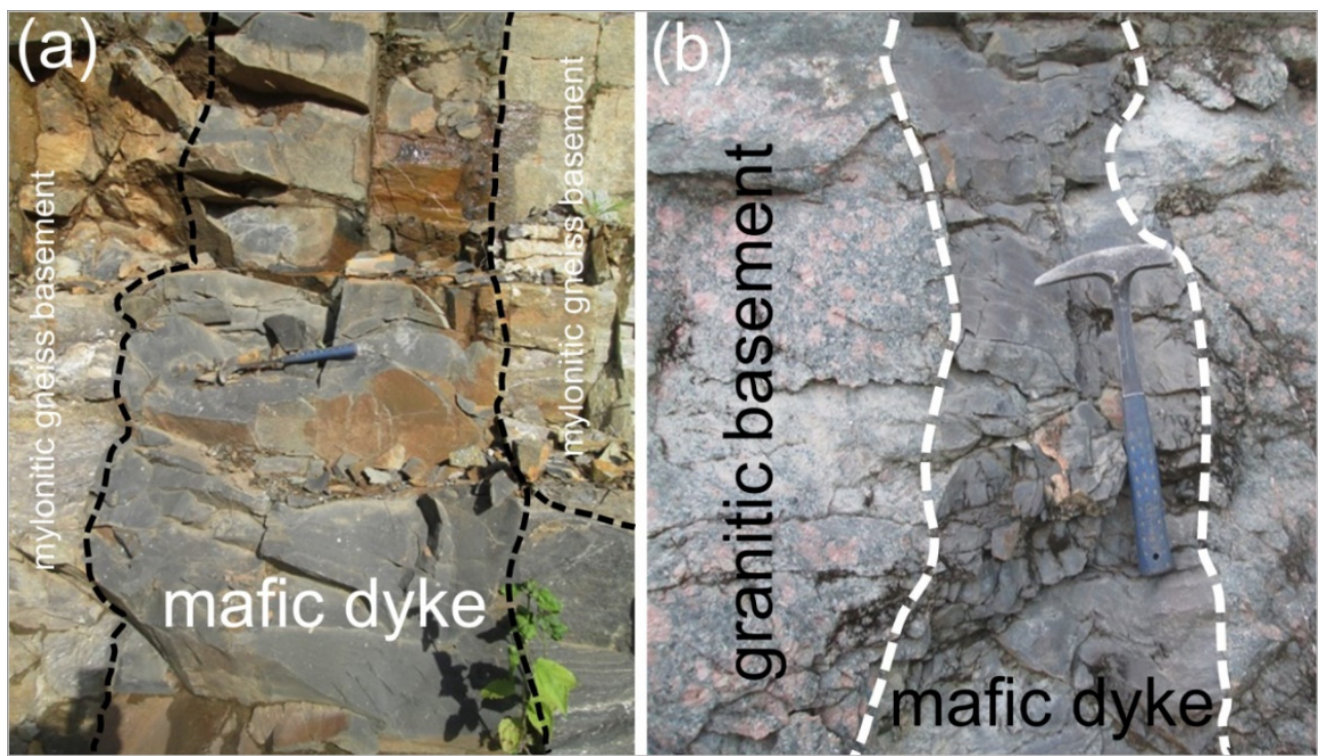

Figure 3. Field geology of Njimom area. $(\mathbf{a}, \mathbf{b})$ Dykes cross-cutting the Precambrian basement (mylonitic gneiss) in (a) and (granite) in (b). 
The Njimom dykes that intrude the mylonitic basement show a weakly porphyritic to aphyric texture (Figure $4 \mathrm{a}-\mathrm{c}$ ). The groundmass of the dykes NK0 and NK2A is strongly altered (Figure 4a,b). Alteration effects are seen mostly in the presence of clay minerals, chlorite, quartz and calcite in the interstices, and iddingsitization of olivine phenocrysts in the sample NK2A (Figure 4b). The dyke NK2C shows an intergranular texture and contain altered olivine, clinopyroxene, plagioclase, and Fe-Ti oxides (Figure 4c). The dyke NK6 that intrudes the granites has phenocrysts and microlites of corroded olivine, with plagioclase, clinopyroxene, and Fe-Ti oxides in the groundmass. The mesostasis is altered and also has scarce alkali feldspar. Calcite-filled amygdales are common (Figure $4 \mathrm{~d}$ ).

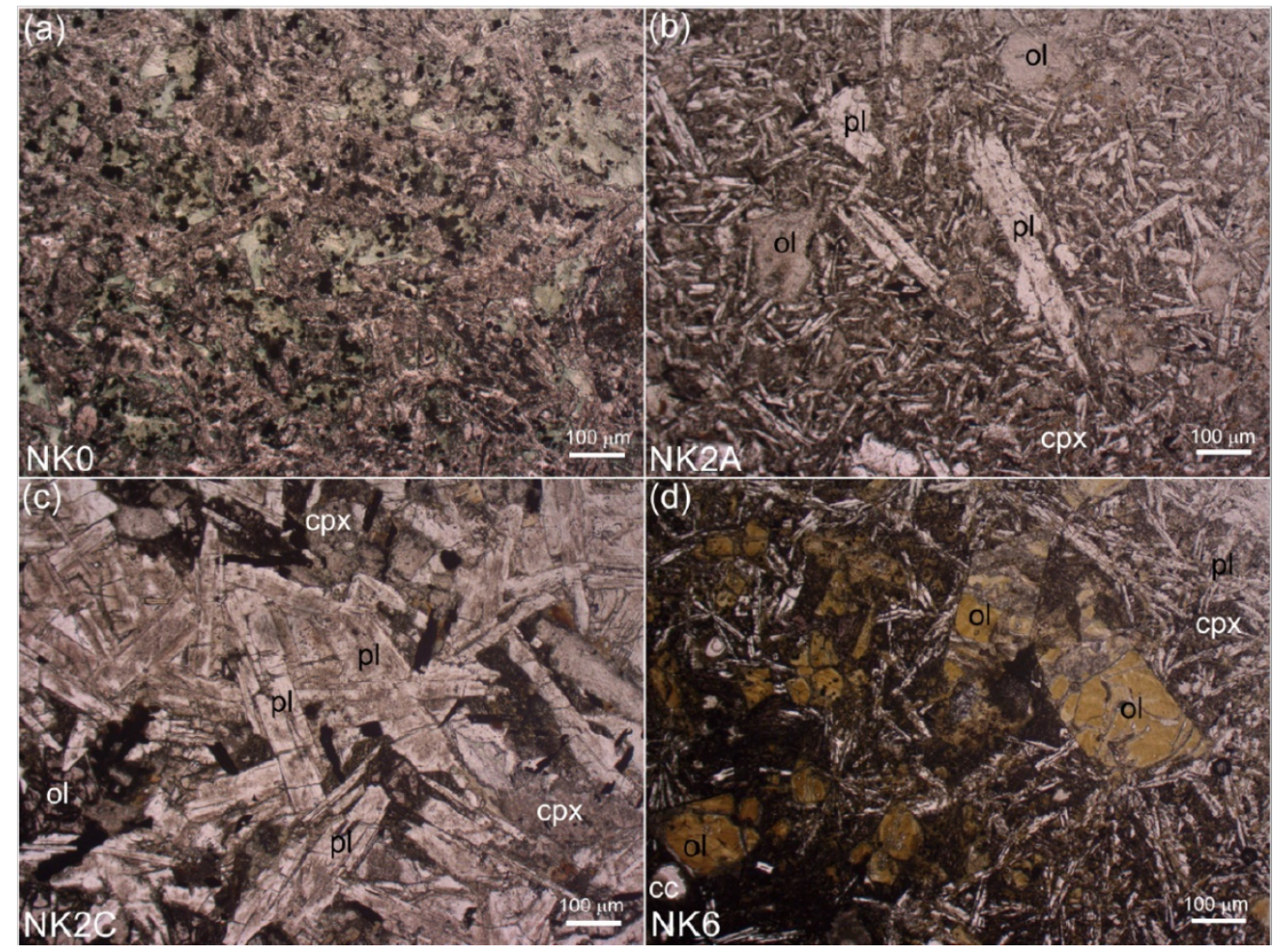

Figure 4. Main petrographic features of the Njimom dykes. (a) Altered aphyric basalt NK0; (b) sub-ophitic texture in basalt NK2A. The olivine phenocrysts are completely altered; (c) intergranular texture in aphyric basalt NK2C with plagioclase, altered olivine, clinopyroxene and opaque oxides; (d) porphyric texture in basalt NK6. The olivine is completely iddingsitized. The dykes in (a-c) cross-cut the mylonitic gneiss. The dyke NK6 intrudes (d) the granitic basement. Mineral names are abbreviated as cpx clinopyroxene; pl, plagioclase; ol, olivine; cc, calcite.

\section{Analytical Methods}

Four dykes of the Njimom area, were chosen for mineral chemical analysis. The mineral compositions (Tables 1-3) were obtained at the University of Naples, using an Oxford Instruments Microanalysis Unit equipped with an INCA X-act detector and a JEOL JSM-5310 microscope in energy-dispersive spectrometry (EDS). The standard operating conditions included a primary beam voltage of $15 \mathrm{kV}$, filament current of 50-100 $\mu \mathrm{A}$ and spot size of 5-10 $\mu \mathrm{m}, 20 \mathrm{~mm}$ WD. Measurements were made with an INCA X-stream pulse processor and with the Energy software. The quant optimization is carried out using cobalt (FWHM-full width at half maximum peak height—of the strobed zero $=60-65 \mathrm{eV}$ ). The following standards were used for calibration: anorthite (Ca), San Carlos olivine (Mg), anorthoclase $(\mathrm{Si}, \mathrm{Al}, \mathrm{Na})$, albite $(\mathrm{Na})$, rutile $(\mathrm{Ti})$, fayalite $(\mathrm{Fe})$, chromite $(\mathrm{Cr})$, serandite $(\mathrm{Mn})$, microcline $(\mathrm{K})$, Durango apatite $(\mathrm{P})$, fluorite $(\mathrm{F})$, barite $(\mathrm{Ba})$, strontianite $(\mathrm{Sr})$, zircon $(\mathrm{Zr}, \mathrm{Hf})$, synthetic Smithsonian orthophosphates ( $\mathrm{La}, \mathrm{Ce}, \mathrm{Nd}, \mathrm{Sm}, \mathrm{Y})$, pure vanadium and niobium $(\mathrm{V}, \mathrm{Nb})$, Corning glass (Th and $\mathrm{U})$, sphalerite $(\mathrm{Zn})$, pyrite $(\mathrm{S})$, galena $(\mathrm{Pb})$, sodium chloride 
$(\mathrm{Cl})$, and pollucite (Cs). The $\mathrm{K} \alpha, \mathrm{L} \alpha$, or $\mathrm{M} \alpha$ lines were used for calibration, according to the element. Further analytical details can be found in [6].

Fourteen samples from the Njimom area were analyzed for whole-rock major and trace element (including rare earth element) concentrations at Activation Laboratories (Actlabs), Ancaster, Ontario (Canada). Rock powders were mixed with fluxes lithium metaborate and lithium tetraborate and fused in an induction furnace. The molten samples were immediately poured into a solution of $5 \%$ nitric acid containing an internal standard and mixed continuously until completely dissolved ( $30 \mathrm{~min})$. The samples were analyzed for major oxides and selected trace elements (Ba, Be, Sc, Sr, V, Y, and $\mathrm{Zr}$ ) on a combination simultaneous/sequential Thermo Jarrell-Ash ENVIRO II ICP or a Varian Vista 735 ICP. Calibration was performed using seven USGS and CANMET certified reference materials. One of the seven standards was used during the analysis for every group of ten samples. The fused sample solutions were diluted and analyzed by a Perkin Elmer Sciex ELAN 6000, 6100 or $9000 \mathrm{ICP} / \mathrm{MS}$ for other trace elements $(\mathrm{Cr}, \mathrm{Co}, \mathrm{Ni}, \mathrm{Cu}, \mathrm{Zn}, \mathrm{Ga}, \mathrm{Ge}, \mathrm{As}, \mathrm{Rb}, \mathrm{Nb}, \mathrm{Mo}$, $\mathrm{Sn}, \mathrm{Cs}, \mathrm{La}, \mathrm{Ce}, \mathrm{Pr}, \mathrm{Nd}, \mathrm{Sm}, \mathrm{Eu}, \mathrm{Gd}, \mathrm{Tb}, \mathrm{Dy}, \mathrm{Ho}, \mathrm{Er}, \mathrm{Tm}, \mathrm{Yb}, \mathrm{Lu}, \mathrm{Hf}, \mathrm{Ta}, \mathrm{W}, \mathrm{Pb}, \mathrm{Bi}, \mathrm{Th}$, and U). Three blanks and five control samples (three before the sample group and two after) were analyzed per group of samples. Duplicates were fused and analyzed after every 10 samples. Analyses of international standards are reported in Table 4. The weight loss on ignition was determined with gravimetric techniques, by firing at $1000^{\circ} \mathrm{C}$ small aliquots of powders previously dried at $110{ }^{\circ} \mathrm{C}$ overnight. Major and trace element (including the rare earth element) data are presented in Table 4. 
Table 1. Representative chemical analyses (in wt.\%) and structural formulas of clinopyroxenes of Njimom dykes (western Cameroon).

\begin{tabular}{|c|c|c|c|c|c|c|c|c|c|c|c|c|c|}
\hline $\begin{array}{l}\text { Sample } \\
\text { Group } \\
\text { Description }\end{array}$ & $\begin{array}{l}\text { NK0 } \\
\text { dyke in } \\
\text { mylonites } \\
\text { gm }\end{array}$ & $\begin{array}{l}\text { NK0 } \\
\text { dyke in } \\
\text { mylonites } \\
\text { gm }\end{array}$ & $\begin{array}{l}\text { NK0 } \\
\text { dyke in } \\
\text { mylonites } \\
\text { gm }\end{array}$ & $\begin{array}{l}\text { NK0 } \\
\text { dyke in } \\
\text { mylonites } \\
\text { gm }\end{array}$ & $\begin{array}{l}\text { NK0 } \\
\text { dyke in } \\
\text { mylonites } \\
\text { gm }\end{array}$ & $\begin{array}{l}\text { NK0 } \\
\text { dyke in } \\
\text { mylonites } \\
\text { gm }\end{array}$ & $\begin{array}{c}\text { NK0 } \\
\text { dyke in } \\
\text { mylonites } \\
\text { gm }\end{array}$ & $\begin{array}{l}\text { NK0 } \\
\text { dyke in } \\
\text { mylonites } \\
\text { gm }\end{array}$ & $\begin{array}{c}\text { NK0 } \\
\text { dyke in } \\
\text { mylonites } \\
\text { gm }\end{array}$ & $\begin{array}{l}\text { NK0 } \\
\text { dyke in } \\
\text { mylonites } \\
\text { gm }\end{array}$ & $\begin{array}{l}\text { NK0 } \\
\text { dyke in } \\
\text { mylonites } \\
\text { gm }\end{array}$ & $\begin{array}{l}\text { NK2C } \\
\text { dyke in } \\
\text { mylonites } \\
\text { gm }\end{array}$ & $\begin{array}{c}\text { NK2C } \\
\text { dyke in } \\
\text { mylonites } \\
\text { gm }\end{array}$ \\
\hline $\mathrm{TiO}_{2}$ & 2.35 & 1.13 & 1.10 & 3.29 & 0.17 & 0.05 & 1.25 & 1.59 & 0.93 & 1.22 & 1.58 & 2.54 & 2.77 \\
\hline $\mathrm{Al}_{2} \mathrm{O}_{3}$ & 6.35 & 2.48 & 2.41 & 6.79 & 1.33 & 1.38 & 2.48 & 4.63 & 1.79 & 4.08 & 5.20 & 5.84 & 5.84 \\
\hline $\mathrm{FeO}$ & 10.75 & 10.29 & 10.00 & 10.32 & 18.63 & 17.37 & 9.90 & 12.09 & 12.75 & 12.40 & 10.16 & 9.44 & 9.23 \\
\hline $\mathrm{MnO}$ & - & 0.73 & 0.23 & 0.08 & 0.89 & 0.80 & 0.32 & 0.46 & 1.13 & 0.90 & 0.54 & 0.36 & - \\
\hline $\mathrm{CaO}$ & 21.75 & 21.20 & 21.73 & 21.33 & 21.26 & 21.91 & 21.02 & 21.04 & 21.31 & 20.91 & 20.93 & 20.40 & 20.21 \\
\hline $\mathrm{Na}_{2} \mathrm{O}$ & 0.50 & 0.15 & 0.51 & 0.59 & 0.92 & 1.01 & 0.40 & 0.82 & 0.40 & 0.49 & 0.52 & 0.53 & 0.58 \\
\hline $\mathrm{Cr}_{2} \mathrm{O}_{3}$ & 0.15 & - & - & 0.13 & - & 0.25 & - & 0.10 & 0.06 & 0.13 & - & - & 0.12 \\
\hline $\mathrm{V}_{2} \mathrm{O}_{3}$ & 0.08 & - & 0.06 & 0.17 & 0.10 & 0.15 & 0.29 & - & - & 0.10 & 0.01 & 0.32 & - \\
\hline sum & 101.45 & 97.86 & 99.42 & 101.53 & 101.63 & 101.48 & 98.76 & 99.74 & 100.08 & 99.28 & 98.51 & 99.06 & 97.59 \\
\hline Mg\# & 66 & 68 & 69 & 67 & 43 & 45 & 70 & 63 & 62 & 59 & 67 & 71 & 71 \\
\hline $\mathrm{Si}$ & 1.768 & 1.897 & 1.912 & 1.743 & 1.929 & 1.928 & 1.913 & 1.799 & 1.893 & 1.876 & 1.818 & 1.760 & 1.762 \\
\hline $\mathrm{Al}^{\mathrm{IV}}$ & 0.218 & 0.097 & 0.081 & 0.246 & 0.048 & 0.048 & 0.085 & 0.174 & 0.089 & 0.120 & 0.169 & 0.222 & 0.223 \\
\hline $\mathrm{Ti}$ & 0.066 & 0.033 & 0.031 & 0.092 & 0.005 & 0.001 & 0.036 & 0.045 & 0.027 & 0.035 & 0.045 & 0.072 & 0.080 \\
\hline $\mathrm{Fe}^{3+}$ & 0.000 & 0.000 & 0.000 & 0.000 & 0.000 & 0.000 & 0.000 & 0.000 & 0.000 & 0.000 & 0.000 & 0.000 & 0.000 \\
\hline $\mathrm{Fe}^{2+}$ & 0.333 & 0.331 & 0.315 & 0.319 & 0.595 & 0.553 & 0.315 & 0.382 & 0.404 & 0.397 & 0.323 & 0.298 & 0.295 \\
\hline $\mathrm{Mn}$ & 0.000 & 0.024 & 0.007 & 0.003 & 0.029 & 0.026 & 0.010 & 0.015 & 0.036 & 0.029 & 0.018 & 0.012 & 0.000 \\
\hline $\mathrm{Mg}$ & 0.650 & 0.719 & 0.710 & 0.650 & 0.444 & 0.449 & 0.721 & 0.639 & 0.664 & 0.576 & 0.670 & 0.727 & 0.727 \\
\hline $\mathrm{Ca}$ & 0.863 & 0.873 & 0.878 & 0.846 & 0.869 & 0.894 & 0.855 & 0.851 & 0.865 & 0.859 & 0.854 & 0.824 & 0.827 \\
\hline $\mathrm{Na}$ & 0.036 & 0.011 & 0.037 & 0.042 & 0.068 & 0.074 & 0.030 & 0.060 & 0.030 & 0.036 & 0.039 & 0.038 & 0.043 \\
\hline $\mathrm{Cr}$ & 0.005 & 0.000 & 0.000 & 0.004 & 0.000 & 0.008 & 0.000 & 0.003 & 0.002 & 0.004 & 0.000 & 0.000 & 0.004 \\
\hline V & 0.002 & 0.000 & 0.002 & 0.005 & 0.003 & 0.005 & 0.009 & 0.000 & 0.000 & 0.003 & 0.000 & 0.010 & 0.000 \\
\hline sum & 4.000 & 4.000 & 4.000 & 4.000 & 4.000 & 4.000 & 4.000 & 4.000 & 4.000 & 4.000 & 4.000 & 4.000 & 4.000 \\
\hline $\mathrm{Ca}$ & 47 & 45 & 46 & 47 & 45 & 47 & 45 & 45 & 44 & 46 & 46 & 44 & 45 \\
\hline $\mathrm{Fe}^{*}$ & 18 & 18 & 17 & 18 & 32 & 30 & 17 & 21 & 22 & 23 & 18 & 17 & 16 \\
\hline $\mathrm{Mg}$ & 35 & 37 & 37 & 36 & 23 & 23 & 38 & 34 & 34 & 31 & 36 & 39 & 39 \\
\hline
\end{tabular}

$\mathrm{gm}=$ groundmass $; \mathrm{Mg} \#=$ atomic $100 \times \mathrm{Mg} /(\mathrm{Mg}+\mathrm{Fe})$.

$\mathrm{Ca}, \mathrm{Fe}^{*}(\mathrm{Fe}+\mathrm{Mn})$ and $\mathrm{Mg}$ in $\mathrm{mol} \%$. 
Table 1. Cont.

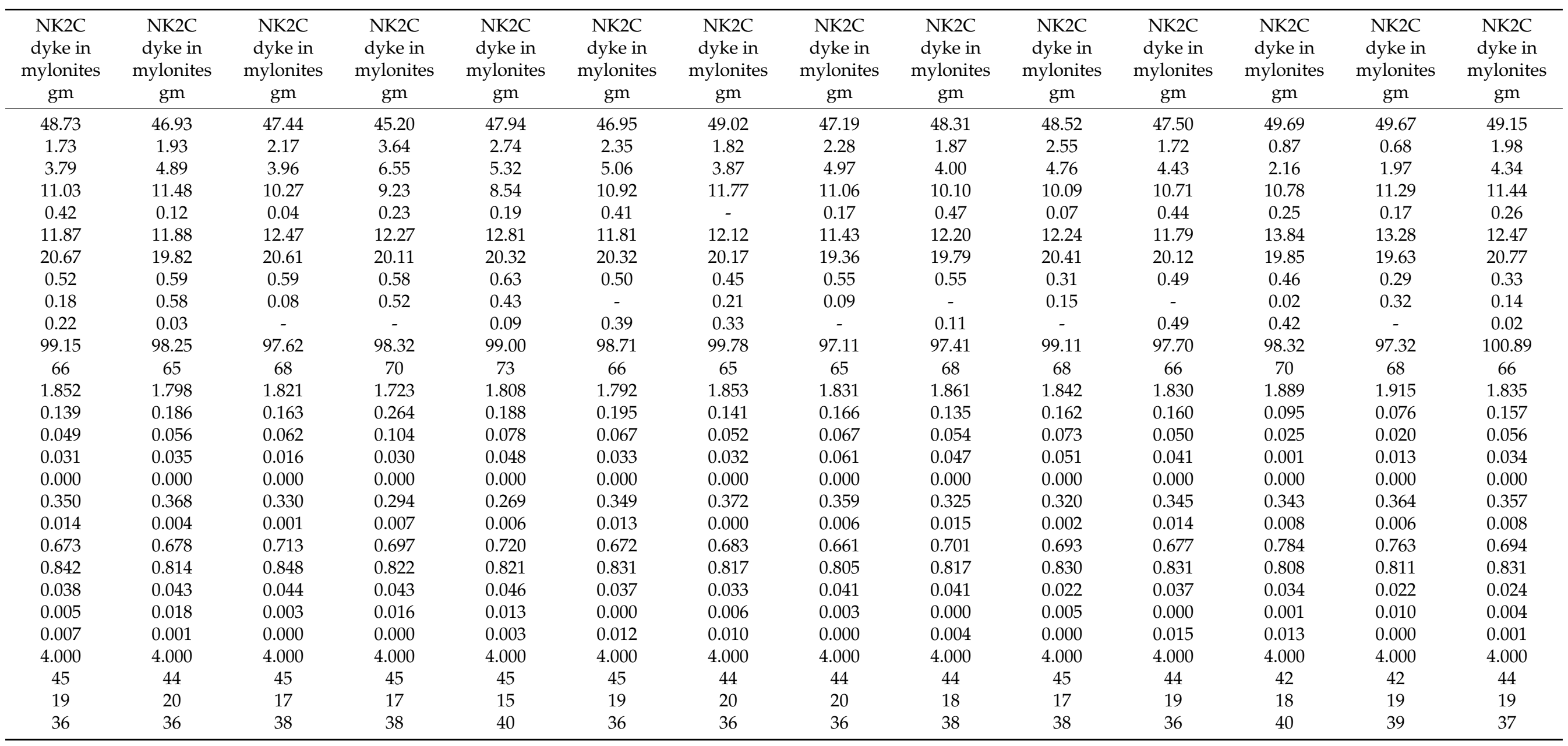


Table 1. Cont.

\begin{tabular}{|c|c|c|c|c|c|c|c|c|c|c|c|c|}
\hline $\begin{array}{c}\text { NK2C } \\
\text { dyke in } \\
\text { mylonites } \\
\text { gm }\end{array}$ & $\begin{array}{c}\text { NK6 } \\
\text { dyke in } \\
\text { granites } \\
\text { gm }\end{array}$ & $\begin{array}{l}\text { NK6 } \\
\text { dyke in } \\
\text { granites } \\
\text { gm }\end{array}$ & $\begin{array}{c}\text { NK6 } \\
\text { dyke in } \\
\text { granites } \\
\text { gm }\end{array}$ & $\begin{array}{c}\text { NK6 } \\
\text { dyke in } \\
\text { granites } \\
\text { gm }\end{array}$ & $\begin{array}{c}\text { NK6 } \\
\text { dyke in } \\
\text { granites } \\
\text { gm }\end{array}$ & $\begin{array}{c}\text { NK6 } \\
\text { dyke in } \\
\text { granites } \\
\text { gm }\end{array}$ & $\begin{array}{l}\text { NK6 } \\
\text { dyke in } \\
\text { granites } \\
\text { gm }\end{array}$ & $\begin{array}{c}\text { NK6 } \\
\text { dyke in } \\
\text { granites } \\
\text { gm }\end{array}$ & $\begin{array}{l}\text { NK6 } \\
\text { dyke in } \\
\text { granites } \\
\text { gm }\end{array}$ & $\begin{array}{c}\text { NK6 } \\
\text { dyke in } \\
\text { granites } \\
\text { gm }\end{array}$ & $\begin{array}{c}\text { NK6 } \\
\text { dyke in } \\
\text { granites } \\
\text { gm }\end{array}$ & $\begin{array}{c}\text { NK6 } \\
\text { dyke in } \\
\text { granites } \\
\text { gm }\end{array}$ \\
\hline 2.02 & 6.10 & 5.56 & 6.58 & 5.28 & 5.49 & 5.42 & 6.12 & 6.36 & 6.67 & 7.24 & 5.39 & 6.76 \\
\hline 5.13 & 8.40 & 8.15 & 9.15 & 8.82 & 8.73 & 6.79 & 6.97 & 8.36 & 7.33 & 8.30 & 7.34 & 9.27 \\
\hline 9.23 & 12.02 & 11.54 & 10.73 & 10.76 & 10.81 & 14.00 & 13.74 & 13.08 & 11.67 & 11.23 & 11.28 & 12.93 \\
\hline 0.12 & 0.19 & 0.04 & - & - & 0.17 & 0.35 & - & 0.17 & 0.33 & 0.11 & 0.05 & - \\
\hline 20.85 & 21.04 & 21.38 & 20.95 & 22.35 & 21.69 & 22.15 & 21.61 & 21.34 & 20.81 & 21.19 & 20.68 & 21.54 \\
\hline 0.57 & 0.61 & 0.93 & 0.50 & 0.53 & 0.61 & 0.58 & 0.43 & 0.87 & 0.49 & 0.88 & 0.57 & 0.67 \\
\hline 0.00 & - & - & - & - & 0.31 & 0.06 & 0.56 & - & 0.24 & 0.11 & 0.41 & 0.22 \\
\hline 0.49 & 0.08 & 0.28 & 0.61 & - & 0.30 & 0.19 & 0.29 & 0.11 & 0.03 & 0.08 & 0.13 & 0.22 \\
\hline 97.72 & 99.75 & 99.09 & 98.05 & 99.52 & 100.59 & 100.46 & 100.94 & 100.93 & 96.04 & 99.63 & 96.44 & 101.42 \\
\hline 70 & 56 & 58 & 58 & 61 & 63 & 50 & 51 & 54 & 56 & 59 & 57 & 54 \\
\hline 1.797 & 1.636 & 1.632 & 1.608 & 1.612 & 1.595 & 1.66 & 1.66 & 1.60 & 1.61 & 1.59 & 1.67 & 1.56 \\
\hline 0.189 & 0.357 & 0.350 & 0.393 & 0.369 & 0.382 & 0.32 & 0.33 & 0.38 & 0.38 & 0.39 & 0.32 & 0.42 \\
\hline 0.058 & 0.176 & 0.161 & 0.194 & 0.151 & 0.156 & 0.16 & 0.18 & 0.18 & 0.20 & 0.21 & 0.16 & 0.19 \\
\hline 0.295 & 0.386 & 0.371 & 0.351 & 0.344 & 0.341 & 0.45 & 0.44 & 0.42 & 0.39 & 0.36 & 0.37 & 0.41 \\
\hline 0.004 & 0.006 & 0.001 & 0.000 & 0.000 & 0.006 & 0.01 & 0.00 & 0.01 & 0.01 & 0.00 & 0.00 & 0.00 \\
\hline 0.702 & 0.499 & 0.505 & 0.491 & 0.544 & 0.574 & 0.45 & 0.45 & 0.48 & 0.50 & 0.52 & 0.50 & 0.48 \\
\hline 0.854 & 0.866 & 0.882 & 0.878 & 0.914 & 0.877 & 0.91 & 0.89 & 0.87 & 0.89 & 0.87 & 0.88 & 0.87 \\
\hline 0.043 & 0.046 & 0.069 & 0.038 & 0.039 & 0.045 & 0.04 & 0.03 & 0.06 & 0.04 & 0.07 & 0.04 & 0.05 \\
\hline 0.000 & 0.000 & 0.000 & 0.000 & 0.000 & 0.009 & 0.00 & 0.02 & 0.00 & 0.01 & 0.00 & 0.01 & 0.01 \\
\hline 0.015 & 0.003 & 0.008 & 0.019 & 0.000 & 0.009 & 0.01 & 0.01 & 0.00 & 0.00 & 0.00 & 0.00 & 0.01 \\
\hline 4.000 & 4.000 & 4.000 & 4.000 & 4.000 & 4.000 & 4.00 & 4.00 & 4.00 & 4.00 & 4.00 & 4.00 & 4.00 \\
\hline 46 & 49 & 50 & 51 & 51 & 49 & 50 & 50 & 49 & 50 & 50 & 50 & 50 \\
\hline 16 & 22 & 21 & 20 & 19 & 19 & 25 & 25 & 24 & 22 & 21 & 21 & 23 \\
\hline 38 & 28 & 29 & 29 & 30 & 32 & 25 & 25 & 27 & 28 & 30 & 29 & 27 \\
\hline
\end{tabular}


Table 2. Representative chemical analyses (in wt.\%) and structural formulas of feldspars of Njimom dykes (western Cameroon).

\begin{tabular}{|c|c|c|c|c|c|c|c|c|c|c|c|}
\hline $\begin{array}{l}\text { Sample } \\
\text { Group }\end{array}$ & $\begin{array}{c}\text { NK2A } \\
\text { dyke in } \\
\text { mylonites }\end{array}$ & $\begin{array}{c}\text { NK2A } \\
\text { dyke in } \\
\text { mylonites }\end{array}$ & $\begin{array}{c}\text { NK2A } \\
\text { dyke in } \\
\text { mylonites }\end{array}$ & $\begin{array}{c}\text { NK2A } \\
\text { dyke in } \\
\text { mylonites }\end{array}$ & $\begin{array}{c}\text { NK2A } \\
\text { dyke in } \\
\text { mylonites }\end{array}$ & $\begin{array}{c}\text { NK2A } \\
\text { dyke in } \\
\text { mylonites }\end{array}$ & $\begin{array}{c}\text { NK2A } \\
\text { dyke in } \\
\text { mylonites }\end{array}$ & $\begin{array}{c}\text { NK2A } \\
\text { dyke in } \\
\text { mylonites }\end{array}$ & $\begin{array}{c}\text { NK2A } \\
\text { dyke in } \\
\text { mylonites }\end{array}$ & $\begin{array}{c}\text { NK2A } \\
\text { dyke in } \\
\text { mylonites }\end{array}$ & $\begin{array}{c}\text { NK2A } \\
\text { dyke in } \\
\text { mylonites }\end{array}$ \\
\hline $\begin{array}{l}\text { Mineral } \\
\text { Name }\end{array}$ & $\mathrm{pl}$ & $\mathrm{pl}$ & $\mathrm{pl}$ & $\mathrm{pl}$ & $\mathrm{pl}$ & $\mathrm{pl}$ & $\mathrm{pl}$ & $\mathrm{pl}$ & $\mathrm{pl}$ & $\mathrm{pl}$ & $\mathrm{pl}$ \\
\hline Description & gm & $\mathrm{gm}$ & $\mathrm{gm}$ & gm & $\mathrm{gm}$ & $\mathrm{gm}$ & $\mathrm{gm}$ & gm & $\mathrm{gm}$ & $\mathrm{gm}$ & $\mathrm{gm}$ \\
\hline $\mathrm{SiO}_{2}$ & 53.45 & 52.66 & 53.71 & 51.39 & 53.49 & 53.99 & 52.37 & 54.22 & 53.26 & 52.94 & 56.05 \\
\hline $\mathrm{Al}_{2} \mathrm{O}_{3}$ & 29.29 & 27.93 & 30.20 & 30.49 & 29.59 & 30.11 & 28.59 & 29.53 & 29.17 & 27.69 & 27.71 \\
\hline $\mathrm{FeO}$ & 0.75 & 0.62 & 0.45 & 0.05 & 0.79 & 0.55 & 0.31 & 0.24 & 0.80 & 0.89 & 0.47 \\
\hline $\mathrm{Na}_{2} \mathrm{O}$ & 4.59 & 4.09 & 4.11 & 3.98 & 4.46 & 4.32 & 4.24 & 4.48 & 4.52 & 4.11 & 5.21 \\
\hline $\mathrm{K}_{2} \mathrm{O}$ & 0.55 & 0.36 & 0.36 & 0.43 & 0.55 & 0.47 & 0.59 & 0.44 & 0.65 & 0.64 & 0.61 \\
\hline $\mathrm{BaO}$ & - & 0.05 & - & - & - & 0.46 & 0.21 & 0.22 & 0.09 & 0.17 & - \\
\hline sum & 100.1 & 97.2 & 100.8 & 98.8 & 100.9 & 101.9 & 97.9 & 100.7 & 100.1 & 97.7 & 99.7 \\
\hline An & 56 & 59 & 60 & 62 & 58 & 58 & 58 & 57 & 56 & 58 & 49 \\
\hline $\mathrm{Ab}$ & 41 & 38 & 38 & 36 & 39 & 38 & 38 & 40 & 40 & 38 & 48 \\
\hline Or & 3 & 2 & 2 & 3 & 3 & 4 & 4 & 3 & 4 & 4 & 4 \\
\hline $\mathrm{Si}$ & 9.675 & 9.858 & 9.686 & 9.434 & 9.611 & 9.642 & 9.718 & 9.774 & 9.652 & 9.873 & 10.158 \\
\hline $\mathrm{Al}$ & 6.250 & 6.166 & 6.420 & 6.600 & 6.268 & 6.338 & 6.254 & 6.276 & 6.231 & 6.088 & 5.921 \\
\hline $\mathrm{Ca}$ & 2.221 & 2.305 & 2.306 & 2.443 & 2.321 & 2.301 & 2.301 & 2.231 & 2.250 & 2.250 & 1.877 \\
\hline $\mathrm{Na}$ & 1.612 & 1.485 & 1.436 & 1.416 & 1.554 & 1.497 & 1.525 & 1.566 & 1.588 & 1.485 & 1.831 \\
\hline K & 0.128 & 0.085 & 0.083 & 0.100 & 0.127 & 0.107 & 0.139 & 0.101 & 0.151 & 0.151 & 0.142 \\
\hline $\mathrm{Ba}$ & 0.000 & 0.003 & 0.000 & 0.000 & 0.000 & 0.033 & 0.015 & 0.016 & 0.007 & 0.012 & 0.000 \\
\hline sum & 20.000 & 20.000 & 20.000 & 20.000 & 20.000 & 20.000 & 20.000 & 20.000 & 20.000 & 20.000 & 20.000 \\
\hline
\end{tabular}


Table 2. Cont.

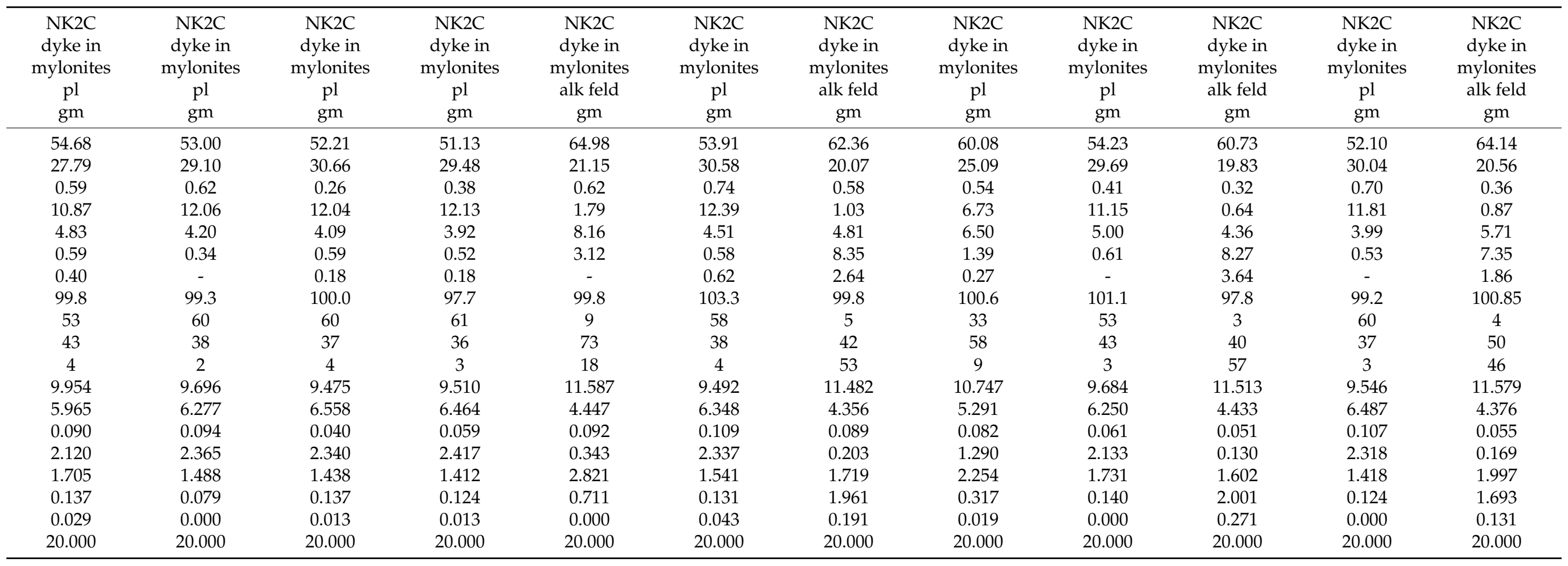


Table 2. Cont.

\begin{tabular}{|c|c|c|c|c|c|c|c|c|c|c|c|}
\hline $\begin{array}{c}\text { NK2C } \\
\text { dyke in } \\
\text { mylonites } \\
\text { pl } \\
\text { gm }\end{array}$ & $\begin{array}{c}\text { NK6 } \\
\text { dyke in } \\
\text { granites } \\
\text { pl } \\
\text { gm }\end{array}$ & $\begin{array}{l}\text { NK6 } \\
\text { dyke in } \\
\text { granites } \\
\text { alk feld } \\
\text { gm }\end{array}$ & $\begin{array}{c}\text { NK6 } \\
\text { dyke in } \\
\text { granites } \\
\text { pl } \\
\text { gm }\end{array}$ & $\begin{array}{c}\text { NK6 } \\
\text { dyke in } \\
\text { granites } \\
\text { pl } \\
\text { gm }\end{array}$ & $\begin{array}{c}\text { NK6 } \\
\text { dyke in } \\
\text { granites } \\
\text { pl } \\
\text { gm }\end{array}$ & $\begin{array}{c}\text { NK6 } \\
\text { dyke in } \\
\text { granites } \\
\text { pl } \\
\text { gm }\end{array}$ & $\begin{array}{c}\text { NK6 } \\
\text { dyke in } \\
\text { granites } \\
\text { pl } \\
\text { gm }\end{array}$ & $\begin{array}{c}\text { NK6 } \\
\text { dyke in } \\
\text { granites } \\
\text { pl } \\
\text { gm }\end{array}$ & $\begin{array}{c}\text { NK6 } \\
\text { dyke in } \\
\text { granites } \\
\text { pl } \\
\text { gm }\end{array}$ & $\begin{array}{c}\text { NK6 } \\
\text { dyke in } \\
\text { granites } \\
\text { pl } \\
\text { gm }\end{array}$ & $\begin{array}{c}\text { NK6 } \\
\text { dyke in } \\
\text { granites } \\
\text { pl } \\
\text { gm }\end{array}$ \\
\hline 54.00 & 53.16 & 65.78 & 53.38 & 53.50 & 54.04 & 52.12 & 53.73 & 56.82 & 62.83 & 53.42 & 53.92 \\
\hline 27.45 & 29.44 & 19.04 & 29.30 & 29.42 & 28.14 & 29.97 & 28.73 & 28.92 & 21.58 & 29.31 & 29.16 \\
\hline 0.64 & 0.99 & 0.41 & 0.47 & 0.87 & 0.93 & 1.24 & 0.44 & 0.53 & 0.45 & 1.37 & 0.75 \\
\hline 9.22 & 12.57 & 0.57 & 11.60 & 12.13 & 10.16 & 12.54 & 11.26 & 10.98 & 3.20 & 12.14 & 11.38 \\
\hline 0.71 & 0.11 & 8.76 & 0.41 & 0.23 & 0.30 & 0.40 & 0.49 & 0.50 & 2.39 & 0.34 & 0.41 \\
\hline 0.16 & - & 0.45 & 0.05 & 0.14 & 0.10 & 0.49 & 0.53 & 0.06 & 0.89 & 0.40 & 0.18 \\
\hline 97.46 & 100.54 & 100.50 & 99.57 & 100.47 & 98.48 & 100.76 & 99.96 & 102.86 & 99.58 & 101.27 & 100.12 \\
\hline 47 & 62 & 3 & 58 & 61 & 53 & 61 & 54 & 53 & 15 & 59 & 58 \\
\hline 49 & 38 & 47 & 40 & 38 & 45 & 35 & 42 & 44 & 70 & 38 & 40 \\
\hline 5 & 1 & 50 & 3 & 2 & 2 & 3 & 4 & 3 & 15 & 3 & 3 \\
\hline 10.000 & 9.613 & 11.833 & 9.727 & 9.694 & 9.951 & 9.443 & 9.759 & 10.004 & 11.275 & 9.623 & 9.797 \\
\hline 5.993 & 6.277 & 4.039 & 6.296 & 6.284 & 6.110 & 6.403 & 6.152 & 6.004 & 4.566 & 6.224 & 6.246 \\
\hline 0.099 & 0.149 & 0.062 & 0.072 & 0.131 & 0.142 & 0.188 & 0.066 & 0.078 & 0.067 & 0.207 & 0.113 \\
\hline 0.168 & 0.026 & 2.010 & 0.095 & 0.052 & 0.071 & 0.094 & 0.114 & 0.113 & 0.548 & 0.078 & 0.096 \\
\hline 0.011 & 0.000 & 0.032 & 0.003 & 0.010 & 0.007 & 0.035 & 0.038 & 0.004 & 0.063 & 0.028 & 0.013 \\
\hline 20.000 & 20.000 & 20.000 & 20.000 & 20.000 & 20.000 & 20.000 & 20.000 & 20.000 & 20.000 & 20.000 & 20.000 \\
\hline
\end{tabular}


Table 2. Cont.

\begin{tabular}{|c|c|c|c|}
\hline $\begin{array}{c}\text { NK6 } \\
\text { dyke in } \\
\text { granites } \\
\text { pl } \\
\text { gm }\end{array}$ & $\begin{array}{l}\text { NK6 } \\
\text { dyke in } \\
\text { granites } \\
\text { alk feld } \\
\text { gm }\end{array}$ & $\begin{array}{c}\text { NK6 } \\
\text { dyke in } \\
\text { granites } \\
\text { pl } \\
\text { gm }\end{array}$ & $\begin{array}{l}\text { NK6 } \\
\text { dyke in } \\
\text { granites } \\
\text { alk feld } \\
\text { gm }\end{array}$ \\
\hline 55.74 & 66.85 & 65.47 & 65.34 \\
\hline 29.34 & 20.08 & 21.65 & 20.01 \\
\hline 0.94 & 0.43 & 0.06 & 0.52 \\
\hline 11.95 & 1.49 & 2.37 & 1.78 \\
\hline 0.40 & 3.47 & 2.44 & 3.83 \\
\hline 0.23 & 0.06 & 0.20 & 0.21 \\
\hline 103.21 & 100.90 & 100.36 & 99.33 \\
\hline 57 & 7 & 12 & 9 \\
\hline 40 & 73 & 73 & 68 \\
\hline 3 & 20 & 15 & 23 \\
\hline 9.826 & 11.786 & 11.627 & 11.768 \\
\hline 6.098 & 4.173 & 4.532 & 4.249 \\
\hline 0.139 & 0.063 & 0.009 & 0.078 \\
\hline 0.091 & 0.780 & 0.553 & 0.879 \\
\hline 0.016 & 0.004 & 0.014 & 0.015 \\
\hline 20.000 & 20.000 & 20.000 & 20.000 \\
\hline
\end{tabular}


Table 3. Representative chemical analyses (in wt.\%) and structural formulas of chromiferous spinels, magnetites, and ilmenites of Njimom dykes (western Cameroon).

\begin{tabular}{|c|c|c|c|c|c|c|c|c|c|c|}
\hline $\begin{array}{l}\text { Sample } \\
\text { Group } \\
\text { Mineral Name } \\
\text { Description }\end{array}$ & $\begin{array}{c}\text { NK6 } \\
\text { dyke in } \\
\text { mylonites } \\
\text { cr-sp } \\
\text { incl }\end{array}$ & $\begin{array}{l}\text { NK6 } \\
\text { dyke in } \\
\text { mylonites } \\
\text { cr-sp } \\
\text { incl }\end{array}$ & $\begin{array}{c}\text { NK6 } \\
\text { dyke in } \\
\text { mylonites } \\
\text { cr-sp } \\
\text { incl }\end{array}$ & $\begin{array}{l}\text { NK2C } \\
\text { dyke in } \\
\text { mylonites } \\
\text { mgt } \\
\text { gm }\end{array}$ & $\begin{array}{l}\text { NK2C } \\
\text { dyke in } \\
\text { mylonites } \\
\text { mgt } \\
\text { gm }\end{array}$ & $\begin{array}{l}\text { NK2C } \\
\text { dyke in } \\
\text { mylonites } \\
\text { mgt } \\
\text { gm }\end{array}$ & $\begin{array}{l}\text { NK2C } \\
\text { dyke in } \\
\text { mylonites } \\
\text { mgt } \\
\text { gm }\end{array}$ & $\begin{array}{l}\text { NK6 } \\
\text { dyke in } \\
\text { granites } \\
\text { mgt } \\
\text { gm }\end{array}$ & $\begin{array}{c}\text { NK6 } \\
\text { dyke in } \\
\text { granites } \\
\text { mgt } \\
\text { gm }\end{array}$ & $\begin{array}{l}\text { NK6 } \\
\text { dyke in } \\
\text { granites } \\
\text { mgt } \\
\text { gm }\end{array}$ \\
\hline $\mathrm{TiO}_{2}$ & 3.16 & 12.40 & 3.36 & 24.63 & 21.14 & 25.36 & 19.77 & 20.37 & 20.37 & 20.59 \\
\hline $\mathrm{Al}_{2} \mathrm{O}_{3}$ & 24.30 & 9.90 & 25.06 & 2.06 & 0.16 & 0.00 & 4.23 & 3.83 & 3.06 & 4.41 \\
\hline $\mathrm{FeO}$ & 29.57 & 54.97 & 31.14 & 68.64 & 74.02 & 68.22 & 64.04 & 67.72 & 67.10 & 64.55 \\
\hline $\mathrm{MnO}$ & 0.40 & - & 0.67 & 0.51 & 0.48 & 0.88 & 0.86 & 1.90 & 1.71 & 1.51 \\
\hline $\mathrm{Cr}_{2} \mathrm{O}_{3}$ & 27.18 & 18.30 & 26.58 & 0.20 & 0.71 & 0.55 & 7.21 & 1.66 & 2.89 & 2.97 \\
\hline $\mathrm{V}_{2} \mathrm{O}_{3}$ & 0.67 & 0.55 & 0.86 & 0.66 & 0.59 & 1.10 & 1.05 & 1.03 & 0.80 & 0.59 \\
\hline $\mathrm{NiO}$ & - & - & - & - & - & - & 0.55 & - & 0.56 & 0.99 \\
\hline sum & 96.70 & 99.70 & 99.54 & 96.90 & 97.10 & 96.10 & 97.80 & 96.50 & 96.88 & 96.21 \\
\hline $\mathrm{Fe}_{2} \mathrm{O}_{3} \mathrm{wt} \%$ & 10.65 & 17.14 & 12.08 & 17.28 & 26.48 & 16.82 & 16.77 & 21.51 & 21.47 & 18.73 \\
\hline $\mathrm{FeO} w \mathrm{t} \%$ & 19.98 & 39.55 & 20.27 & 53.09 & 50.19 & 53.09 & 48.95 & 48.37 & 47.77 & 47.70 \\
\hline sum & 97.8 & 101.4 & 100.8 & 98.6 & 99.8 & 97.8 & 99.5 & 98.7 & 99.0 & 98.1 \\
\hline $\mathrm{Ti}$ & 0.599 & 2.567 & 0.619 & 0.697 & 0.601 & 0.732 & 0.550 & 0.573 & 0.572 & 0.579 \\
\hline $\mathrm{Al}$ & 7.225 & 3.212 & 7.227 & 0.091 & 0.007 & 0.000 & 0.184 & 0.169 & 0.135 & 0.194 \\
\hline $\mathrm{Fe}^{2+}$ & 4.215 & 9.103 & 4.148 & 1.670 & 1.585 & 1.703 & 1.513 & 1.513 & 1.492 & 1.491 \\
\hline $\mathrm{Mn}$ & 0.085 & 0.000 & 0.138 & 0.016 & 0.015 & 0.029 & 0.027 & 0.060 & 0.054 & 0.048 \\
\hline $\mathrm{Mg}$ & 4.298 & 1.464 & 4.333 & 0.011 & 0.000 & 0.000 & 0.005 & 0.000 & 0.022 & 0.032 \\
\hline $\mathrm{Cr}$ & 5.420 & 3.983 & 5.142 & 0.006 & 0.021 & 0.017 & 0.211 & 0.049 & 0.085 & 0.088 \\
\hline V & 0.135 & 0.122 & 0.168 & 0.020 & 0.018 & 0.034 & 0.031 & 0.031 & 0.024 & 0.018 \\
\hline $\mathrm{Ni}$ & 0.000 & 0.000 & 0.000 & 0.000 & 0.000 & 0.000 & 0.016 & 0.000 & 0.017 & 0.030 \\
\hline sum & 24.000 & 24.000 & 24.000 & 3.000 & 3.000 & 3.000 & 3.004 & 3.000 & 3.004 & 3.007 \\
\hline Ulv & - & - & - & 77 & 62 & 75 & 81 & 71 & 71 & 76 \\
\hline Ilm & - & - & - & - & - & - & - & - & - & - \\
\hline $\mathrm{Mg} \#$ & 50 & 14 & 51 & - & - & - & - & - & - & - \\
\hline Cr\# & 43 & 55 & 42 & - & - & - & - & - & - & - \\
\hline
\end{tabular}

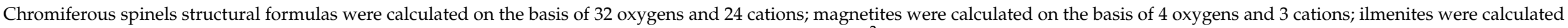
on the basis of 3 oxygens and 2 cations; $\mathrm{Cr} \#=$ atomic $100 \times \mathrm{Cr} /(\mathrm{Cr}+\mathrm{Al}), \mathrm{Mg} \#$ atomic $100 \times \mathrm{Mg} /\left(\mathrm{Mg}+\mathrm{Fe}^{2+}\right)$.

$\mathrm{Ulv}=$ ulvöspinel mol.\%; Ilm = ilmenite mol. \%; gm = groundmass; incl = inclusion; $\mathrm{cr}-\mathrm{sp}=$ chromiferous spinel; $\mathrm{mgt}=\mathrm{magnetite} ; \mathrm{ilm}=\mathrm{ilmenite}$. 
Table 3. Cont.

\begin{tabular}{|c|c|c|c|c|c|c|c|c|c|c|}
\hline $\begin{array}{c}\text { NK6 } \\
\text { dyke in } \\
\text { granites } \\
\text { mgt } \\
\text { gm }\end{array}$ & $\begin{array}{c}\text { NK6 } \\
\text { dyke in } \\
\text { granites } \\
\text { mgt } \\
\text { gm }\end{array}$ & $\begin{array}{c}\text { NK6 } \\
\text { dyke in } \\
\text { granites } \\
\text { mgt } \\
\text { gm }\end{array}$ & $\begin{array}{c}\text { NK6 } \\
\text { dyke in } \\
\text { granites } \\
\text { mgt } \\
\text { gm }\end{array}$ & $\begin{array}{c}\text { NK0 } \\
\text { dyke in } \\
\text { mylonites } \\
\text { ilm } \\
\text { gm }\end{array}$ & $\begin{array}{c}\text { NK0 } \\
\text { dyke in } \\
\text { mylonites } \\
\text { ilm } \\
\text { gm }\end{array}$ & $\begin{array}{l}\text { NK0 } \\
\text { dyke in } \\
\text { mylonites } \\
\text { ilm } \\
\text { gm }\end{array}$ & $\begin{array}{l}\text { NK2A } \\
\text { dyke in } \\
\text { mylonites } \\
\text { ilm } \\
\text { gm }\end{array}$ & $\begin{array}{l}\text { NK2A } \\
\text { dyke in } \\
\text { mylonites } \\
\text { ilm } \\
\text { gm }\end{array}$ & $\begin{array}{l}\text { NK2A } \\
\text { dyke in } \\
\text { mylonites } \\
\text { ilm } \\
\text { gm }\end{array}$ & $\begin{array}{c}\text { NK2A } \\
\text { dyke in } \\
\text { mylonites } \\
\text { ilm } \\
\text { gm }\end{array}$ \\
\hline 24.02 & 22.90 & 26.06 & 24.65 & 49.46 & 51.46 & 48.97 & 47.36 & 49.79 & 50.41 & 50.34 \\
\hline 2.75 & 2.86 & 2.71 & 2.97 & 0.14 & 0.29 & 0.00 & 0.65 & 0.00 & 0.09 & 0.37 \\
\hline 66.83 & 65.87 & 65.65 & 67.52 & 42.94 & 45.06 & 43.89 & 46.73 & 45.81 & 44.56 & 45.35 \\
\hline 1.61 & 2.30 & 1.42 & 1.79 & 3.95 & 4.21 & 4.63 & 1.31 & 1.06 & 2.24 & 1.14 \\
\hline 0.72 & 2.60 & 0.13 & 0.62 & 0.06 & - & - & 0.10 & 0.60 & 0.12 & - \\
\hline 0.36 & 0.60 & 0.85 & - & 0.04 & 0.06 & 0.92 & 0.66 & 0.07 & 0.12 & 0.88 \\
\hline 0.00 & - & 0.54 & - & - & - & - & 0.33 & 0.22 & 0.07 & 0.05 \\
\hline 96.64 & 97.14 & 97.36 & 97.55 & 96.78 & 101.07 & 98.42 & 97.15 & 97.84 & 97.62 & 98.15 \\
\hline 17.35 & 17.39 & 13.20 & 16.74 & 3.07 & 3.36 & 5.04 & 6.07 & 2.90 & 1.65 & 1.43 \\
\hline 51.22 & 50.22 & 53.77 & 52.46 & 40.18 & 42.03 & 39.36 & 41.27 & 43.20 & 43.08 & 44.06 \\
\hline 98.4 & 98.9 & 98.7 & 99.2 & 97.1 & 101.4 & 98.9 & 97.8 & 98.1 & 97.8 & 98.3 \\
\hline 0.678 & 0.645 & 0.735 & 0.691 & 0.967 & 0.964 & 0.942 & 0.921 & 0.964 & 0.980 & 0.971 \\
\hline 0.121 & 0.126 & 0.120 & 0.130 & 0.004 & 0.008 & 0.000 & 0.020 & 0.000 & 0.003 & 0.011 \\
\hline 1.607 & 1.572 & 1.686 & 1.635 & 0.873 & 0.875 & 0.842 & 0.892 & 0.929 & 0.931 & 0.945 \\
\hline 0.051 & 0.073 & 0.045 & 0.056 & 0.087 & 0.089 & 0.100 & 0.029 & 0.023 & 0.049 & 0.025 \\
\hline 0.020 & 0.000 & 0.000 & 0.000 & 0.007 & 0.000 & 0.000 & 0.000 & 0.011 & 0.000 & 0.001 \\
\hline 0.021 & 0.077 & 0.004 & 0.018 & 0.001 & 0.000 & 0.000 & 0.002 & 0.012 & 0.002 & 0.000 \\
\hline 0.011 & 0.018 & 0.025 & 0.000 & 0.001 & 0.001 & 0.019 & 0.014 & 0.001 & 0.003 & 0.018 \\
\hline 0.000 & 0.000 & 0.016 & 0.000 & 0.000 & 0.000 & 0.000 & 0.007 & 0.005 & 0.002 & 0.001 \\
\hline 3.000 & 3.000 & 3.004 & 3.000 & 2.000 & 2.000 & 2.000 & 2.002 & 2.002 & 2.001 & 2.000 \\
\hline 77 & 78 & 84 & 79 & - & - & - & - & - & - & - \\
\hline- & - & - & - & 97 & 97 & 95 & 94 & 97 & 98 & 99 \\
\hline- & - & - & - & - & - & - & - & - & - & - \\
\hline- & - & - & - & - & - & - & - & - & - & - \\
\hline
\end{tabular}


Table 3. Cont.

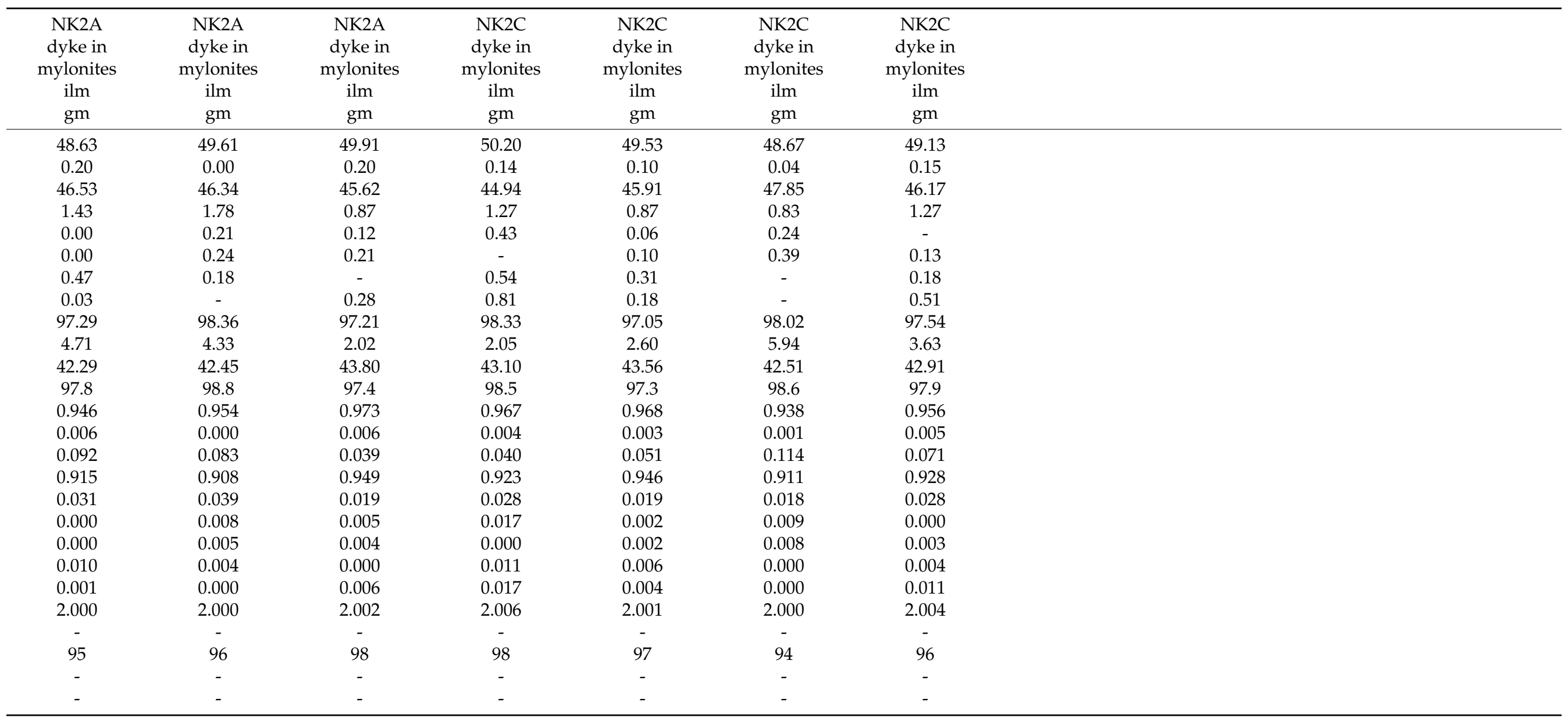


Table 4. Major (wt.\%) and trace (ppm) element data for Njimom dykes (western Cameroon).

\begin{tabular}{|c|c|c|c|c|c|c|c|c|c|c|}
\hline $\begin{array}{l}\text { Sample } \\
\text { Group } \\
\text { Rock Type }\end{array}$ & $\begin{array}{c}\text { NK0 } \\
\text { dyke in } \\
\text { mylonites } \\
\text { haw }\end{array}$ & $\begin{array}{c}\text { NK1 } \\
\text { dyke in } \\
\text { mylonites } \\
\text { haw }\end{array}$ & $\begin{array}{l}\text { NK2A } \\
\text { dyke in } \\
\text { mylonites } \\
\text { alk bas }\end{array}$ & $\begin{array}{c}\text { NK2B } \\
\text { dyke in } \\
\text { mylonites } \\
\text { alk bas }\end{array}$ & $\begin{array}{l}\text { NK2C } \\
\text { dyke in } \\
\text { mylonites } \\
\text { alk bas }\end{array}$ & $\begin{array}{l}\text { NK2D } \\
\text { dyke in } \\
\text { mylonites } \\
\text { alk bas }\end{array}$ & $\begin{array}{l}\mathrm{NK} 2 \mathrm{E} \\
\text { dyke in } \\
\text { mylonites } \\
\text { alk bas }\end{array}$ & $\begin{array}{l}\text { NK2F } \\
\text { dyke in } \\
\text { mylonites } \\
\text { alk bas }\end{array}$ & $\begin{array}{c}\text { NK3 } \\
\text { dyke in } \\
\text { mylonites } \\
\text { haw }\end{array}$ & $\begin{array}{c}\text { NK4 } \\
\text { dyke in } \\
\text { mylonites } \\
\text { alk bas }\end{array}$ \\
\hline $\mathrm{TiO}_{2}$ & 1.75 & 1.73 & 1.90 & 1.78 & 1.91 & 1.85 & 1.94 & 1.93 & 1.67 & 1.60 \\
\hline $\mathrm{Al}_{2} \mathrm{O}_{3}$ & 16.24 & 15.51 & 14.80 & 14.94 & 15.28 & 15.16 & 15.57 & 15.27 & 14.95 & 14.80 \\
\hline $\mathrm{Fe}_{2} \mathrm{O}_{3 \text { tot }}$ & 11.42 & 10.06 & 10.89 & 11.13 & 11.18 & 10.72 & 11.59 & 11.45 & 9.96 & 10.00 \\
\hline $\mathrm{MnO}$ & 0.17 & 0.14 & 0.14 & 0.15 & 0.17 & 0.15 & 0.14 & 0.17 & 0.15 & 0.15 \\
\hline $\mathrm{CaO}$ & 6.16 & 6.15 & 7.98 & 7.33 & 7.37 & 7.36 & 6.59 & 7.57 & 6.43 & 6.79 \\
\hline $\mathrm{Na}_{2} \mathrm{O}$ & 3.70 & 2.49 & 2.71 & 3.03 & 3.29 & 3.35 & 3.08 & 3.12 & 3.11 & 2.65 \\
\hline $\mathrm{K}_{2} \mathrm{O}$ & 2.91 & 3.67 & 1.31 & 1.90 & 2.04 & 1.97 & 1.81 & 1.84 & 2.77 & 2.97 \\
\hline $\mathrm{P}_{2} \mathrm{O}_{5}$ & 0.84 & 0.60 & 0.56 & 0.48 & 0.59 & 0.52 & 0.59 & 0.61 & 0.59 & 0.51 \\
\hline LOI & 3.34 & 4.83 & 6.05 & 4.55 & 5.29 & 6.59 & 4.72 & 5.26 & 4.34 & 4.95 \\
\hline Total & 98.95 & 100.60 & 98.54 & 99.76 & 100.63 & 100.45 & 100.34 & 100.51 & 99.68 & 98.62 \\
\hline $\mathrm{Be}$ & 3 & 2 & 1 & 1 & 2 & 2 & 1 & 1 & 2 & 2 \\
\hline V & 96 & 118 & 149 & 144 & 151 & 148 & 153 & 156 & 119 & 122 \\
\hline $\mathrm{Cr}$ & 70 & 90 & 170 & 170 & 170 & 170 & 180 & 170 & 100 & 100 \\
\hline $\mathrm{Cu}$ & 50 & 40 & 50 & 50 & 50 & 50 & 60 & 50 & 50 & 40 \\
\hline $\mathrm{Zn}$ & 100 & 100 & 100 & 90 & 100 & 90 & 100 & 90 & 110 & 100 \\
\hline $\mathrm{Ga}$ & 18 & 20 & 17 & 17 & 18 & 17 & 18 & 17 & 20 & 20 \\
\hline $\mathrm{Rb}$ & 75 & 122 & 41 & 54 & 53 & 48 & 47 & 54 & 107 & 94 \\
\hline $\mathrm{Sr}$ & 578 & 475 & 437 & 473 & 505 & 513 & 490 & 493 & 449 & 481 \\
\hline $\mathrm{Y}$ & 35 & 34 & 28 & 26 & 28 & 27 & 27 & 29 & 32 & 31 \\
\hline $\mathrm{Zr}$ & 295 & 278 & 211 & 216 & 221 & 209 & 222 & 216 & 286 & 252 \\
\hline $\mathrm{Nb}$ & 51 & 40 & 27 & 26 & 28 & 27 & 28 & 27 & 40 & 35 \\
\hline Sn & 3 & 3 & 2 & 2 & 1 & 2 & 2 & 2 & 3 & 3 \\
\hline Cs & $<0.5$ & 3.6 & 1.4 & 1.2 & 1.2 & 1 & 0.9 & 1.2 & 2.2 & 4 \\
\hline $\mathrm{Ba}$ & 785 & 935 & 402 & 609 & 694 & 710 & 669 & 591 & 784 & 741 \\
\hline $\mathrm{La}$ & 52 & 50.5 & 31 & 30 & 32.1 & 30.3 & 30.5 & 30.2 & 48.4 & 51.8 \\
\hline $\mathrm{Ce}$ & 104 & 104 & 66.4 & 63.9 & 67.7 & 64.9 & 66 & 65.7 & 101 & 107 \\
\hline $\operatorname{Pr}$ & 12.1 & 12.6 & 8.39 & 7.83 & 8.51 & 8.07 & 8.25 & 8.17 & 11.9 & 12.7 \\
\hline $\mathrm{Nd}$ & 46.3 & 47.5 & 33.4 & 31.6 & 34.3 & 32 & 33.1 & 32.7 & 45.3 & 47.7 \\
\hline $\mathrm{Sm}$ & 8.7 & 9.4 & 7.2 & 6.7 & 7.4 & 6.7 & 6.9 & 7 & 9 & 9.7 \\
\hline
\end{tabular}


Table 4. Cont.

\begin{tabular}{|c|c|c|c|c|c|c|c|c|c|c|}
\hline $\mathrm{Eu}$ & 2.29 & 2.13 & 1.95 & 1.91 & 2.02 & 1.96 & 1.87 & 2.01 & 2.12 & 1.93 \\
\hline $\mathrm{Gd}$ & 7.90 & 8.10 & 6.80 & 6.60 & 6.60 & 6.20 & 6.30 & 6.80 & 7.70 & 8.00 \\
\hline $\mathrm{Tb}$ & 1.10 & 1.20 & 1.00 & 0.90 & 1.00 & 1.00 & 1.00 & 1.00 & 1.20 & 1.20 \\
\hline Dy & 6.80 & 7.00 & 6.10 & 4.90 & 6.10 & 5.60 & 5.70 & 6.00 & 6.60 & 7.20 \\
\hline Ho & 1.30 & 1.30 & 1.20 & 1.00 & 1.20 & 1.10 & 1.10 & 1.10 & 1.30 & 1.40 \\
\hline Er & 3.60 & 3.60 & 3.30 & 3.00 & 3.10 & 2.90 & 3.10 & 3.10 & 3.50 & 3.80 \\
\hline $\mathrm{Tm}$ & 0.50 & 0.51 & 0.44 & 0.38 & 0.46 & 0.41 & 0.42 & 0.42 & 0.47 & 0.54 \\
\hline $\mathrm{Yb}$ & 3.30 & 3.40 & 2.80 & 2.50 & 3.00 & 2.60 & 2.90 & 2.90 & 3.10 & 3.40 \\
\hline $\mathrm{Lu}$ & 0.51 & 0.52 & 0.39 & 0.39 & 0.44 & 0.39 & 0.42 & 0.43 & 0.51 & 0.53 \\
\hline $\mathrm{Hf}$ & 6.1 & 6.2 & 4.9 & 4.4 & 4.7 & 4.4 & 4.7 & 4.6 & 6.2 & 6.0 \\
\hline $\mathrm{Pb}$ & 6 & 7 & $<5$ & $<5$ & $<5$ & $<5$ & $<5$ & $<5$ & 6 & 6 \\
\hline Th & 5 & 8 & 2.7 & 2.5 & 2.6 & 2.6 & 2.5 & 2.5 & 7.7 & 9.1 \\
\hline $\mathrm{U}$ & 1.4 & 1.6 & 0.6 & 0.6 & 0.7 & 0.6 & 0.6 & 0.6 & 1.5 & 1.7 \\
\hline
\end{tabular}

Notes: Major oxides are in weight percent (wt.\%) and trace elements in parts per million (ppm). LOI is loss in weight on ignition; alk bas, alkali basalt; haw, hawaiite.

"Cert." are the recommended values and "Meas." the measured values on USGS and CANMET reference materials.

\begin{tabular}{|c|c|c|c|c|c|c|c|c|c|}
\hline $\begin{array}{c}\text { NK5 } \\
\text { dyke in } \\
\text { mylonites } \\
\text { alk bas }\end{array}$ & $\begin{array}{c}\text { NK6 } \\
\text { dyke in } \\
\text { granites } \\
\text { alk bas }\end{array}$ & $\begin{array}{c}\text { NK7 } \\
\text { dyke in } \\
\text { granites } \\
\text { alk bas }\end{array}$ & $\begin{array}{c}\text { NK8 } \\
\text { dyke in } \\
\text { granites } \\
\text { alk bas }\end{array}$ & W-2a Meas & W-2a Cert & SY-4 Meas & SY-4 Cert & JR-1 Meas & JR-1 Cert \\
\hline 51.43 & 42.18 & 41.98 & 43.73 & 52.48 & 52.40 & 49.71 & 49.90 & - & - \\
\hline 1.61 & 2.24 & 2.31 & 2.15 & 1.06 & 1.06 & 0.29 & 0.29 & - & - \\
\hline 15.02 & 12.85 & 13.44 & 12.20 & 15.55 & 15.40 & 20.35 & 20.69 & - & - \\
\hline 9.97 & 10.78 & 10.74 & 12.49 & 10.77 & 10.70 & 6.06 & 6.21 & - & - \\
\hline 0.14 & 0.20 & 0.16 & 0.19 & 0.17 & 0.16 & 0.11 & 0.11 & - & - \\
\hline 5.89 & 13.94 & 11.91 & 8.96 & 11.03 & 10.90 & 8.14 & 8.05 & - & - \\
\hline 2.42 & 2.47 & 2.69 & 2.52 & 2.23 & 2.14 & 6.96 & 7.10 & - & - \\
\hline 2.55 & 0.93 & 1.06 & 0.98 & 0.62 & 0.63 & 1.66 & 1.66 & - & - \\
\hline 0.57 & 0.31 & 0.38 & 0.32 & 0.13 & 0.13 & 0.13 & 0.13 & - & - \\
\hline 4.49 & 9.21 & 9.16 & 8.24 & - & - & - & - & - & - \\
\hline 98.33 & 100.48 & 100.42 & 100.23 & 100.29 & 99.89 & 93.91 & 94.68 & - & - \\
\hline 2 & 2 & 2 & 1 & $<1$ & 1.3 & 3 & 2.6 & - & - \\
\hline 113 & 208 & 215 & 205 & 266 & 262 & 9 & 8 & - & - \\
\hline 90 & 260 & 270 & 280 & 90 & 92 & - & - & - & - \\
\hline
\end{tabular}


Table 4. Cont

\begin{tabular}{|c|c|c|c|c|c|c|c|c|c|}
\hline 40 & 210 & 170 & 230 & 80 & 70 & - & - & $<20$ & 1.67 \\
\hline 40 & 60 & 70 & 60 & 110 & 110 & - & - & $<10$ & 2.68 \\
\hline 100 & 110 & 120 & 100 & 80 & 80 & - & - & 30 & 30.6 \\
\hline 20 & 18 & 19 & 17 & 18 & 17 & - & - & 15 & 16.1 \\
\hline 96 & 18 & 22 & 22 & 20 & 21 & - & - & 252 & 257 \\
\hline 468 & 683 & 608 & 528 & 200 & 190 & 1194 & 1191 & - & - \\
\hline 32 & 18 & 21 & 17 & 20 & 24 & 117 & 119 & - & - \\
\hline 249 & 130 & 128 & 123 & 95 & 94 & 538 & 517 & - & - \\
\hline 35 & 26 & 28 & 25 & - & - & - & - & 15 & 15.2 \\
\hline 3 & 1 & 2 & 1 & - & - & - & - & 3 & 2.86 \\
\hline 4 & 2.8 & 3.5 & 4.9 & - & - & - & - & 20.6 & 20.8 \\
\hline 725 & 268 & 263 & 256 & 173 & 182 & 348 & 340 & - & - \\
\hline 50.6 & 19.3 & 21.8 & 18.1 & 10.1 & 10 & - & - & 19 & 19.7 \\
\hline 105 & 38.1 & 43.7 & 36.7 & 24.3 & 23 & - & - & 44.9 & 47.2 \\
\hline 12.5 & 4.63 & 5.36 & 4.46 & - & - & - & - & 5.6 & 5.58 \\
\hline 47.1 & 18.4 & 21.9 & 18.2 & 13.2 & 13 & - & - & 21.9 & 23.3 \\
\hline 9.3 & 4.2 & 5.3 & 4.2 & 3.4 & 3.3 & - & - & 5.5 & 6.03 \\
\hline 1.89 & 1.58 & 1.79 & 1.51 & - & - & - & - & 0.27 & 0.3 \\
\hline 8.00 & 4.40 & 5.20 & 4.40 & - & - & - & - & 5.5 & 5.06 \\
\hline 1.20 & 0.70 & 0.80 & 0.70 & 0.6 & 0.63 & - & - & 1.1 & 1.01 \\
\hline 1.40 & 0.70 & 0.80 & 0.70 & 0.8 & 0.76 & - & - & - & - \\
\hline 3.70 & 2.00 & 2.20 & 1.90 & - & - & - & - & - & - \\
\hline 0.51 & 0.25 & 0.30 & 0.27 & - & - & - & - & 0.62 & 0.67 \\
\hline 3.30 & 1.50 & 1.80 & 1.60 & 2.1 & 2.1 & - & - & 4.6 & 4.55 \\
\hline 0.50 & 0.23 & 0.25 & 0.23 & 0.31 & 0.33 & - & - & 0.7 & 0.71 \\
\hline 6.0 & 2.9 & 2.9 & 2.8 & - & - & - & - & 4.4 & 4.51 \\
\hline 2.8 & 1.6 & 1.7 & 1.5 & 0.5 & 0.5 & - & - & 2 & 1.86 \\
\hline 6 & $<5$ & $<5$ & $<5$ & - & - & - & - & 18 & 19.3 \\
\hline 9.1 & 2.1 & 2.2 & 2.1 & 2.4 & 2.4 & - & - & 25.4 & 26.7 \\
\hline 1.8 & 0.6 & 0.6 & 0.5 & 0.5 & 0.53 & - & - & 8.6 & 8.88 \\
\hline
\end{tabular}




\section{Results}

\subsection{Mineral Chemistry}

\subsubsection{Pyroxenes}

Clinopyroxene is a typical groundmass phase of Njimom dykes. It is diopside/salite ( $\mathrm{Ca}_{49-51} \mathrm{Fe}_{19-20} \mathrm{Mg}_{29-32}$; Table 1; Figure 5a) in the dyke NK6 that intrudes the Precambrian granites $(\mathrm{Mg \#}=50-59$, where $\mathrm{Mg \#}=$ atomic $\mathrm{Mg} \times 100 /(\mathrm{Mg}+\mathrm{Fe}+\mathrm{Mn})) . \mathrm{TiO}_{2}$ and $\mathrm{Al}_{2} \mathrm{O}_{3}$ contents range from 5.28-6.76 wt.\% and from 6.79 to $9.27 \mathrm{wt} . \%$, respectively (Figure $5 \mathrm{~b}, \mathrm{c}$ ). Clinopyroxene of the dykes (NK0 and NK2C) that intrude the Precambrian mylonitic gneisses is diopside and augite with rare hedenbergite $\left(\mathrm{Ca}_{42-47} \mathrm{Fe}_{18-30} \mathrm{Mg}_{23}-40, \mathrm{Mg} \#=59-73\right.$; Figure 5a). The clinopyroxenes of the dykes NK0 and NK2C have less $\mathrm{TiO}_{2}$ and $\mathrm{Al}_{2} \mathrm{O}_{3}$ contents compared to the clinopyroxenes of the dyke NK6. The clinopyroxene compositions are thus highly distinctive of the different magma types (Figure 5b,c).

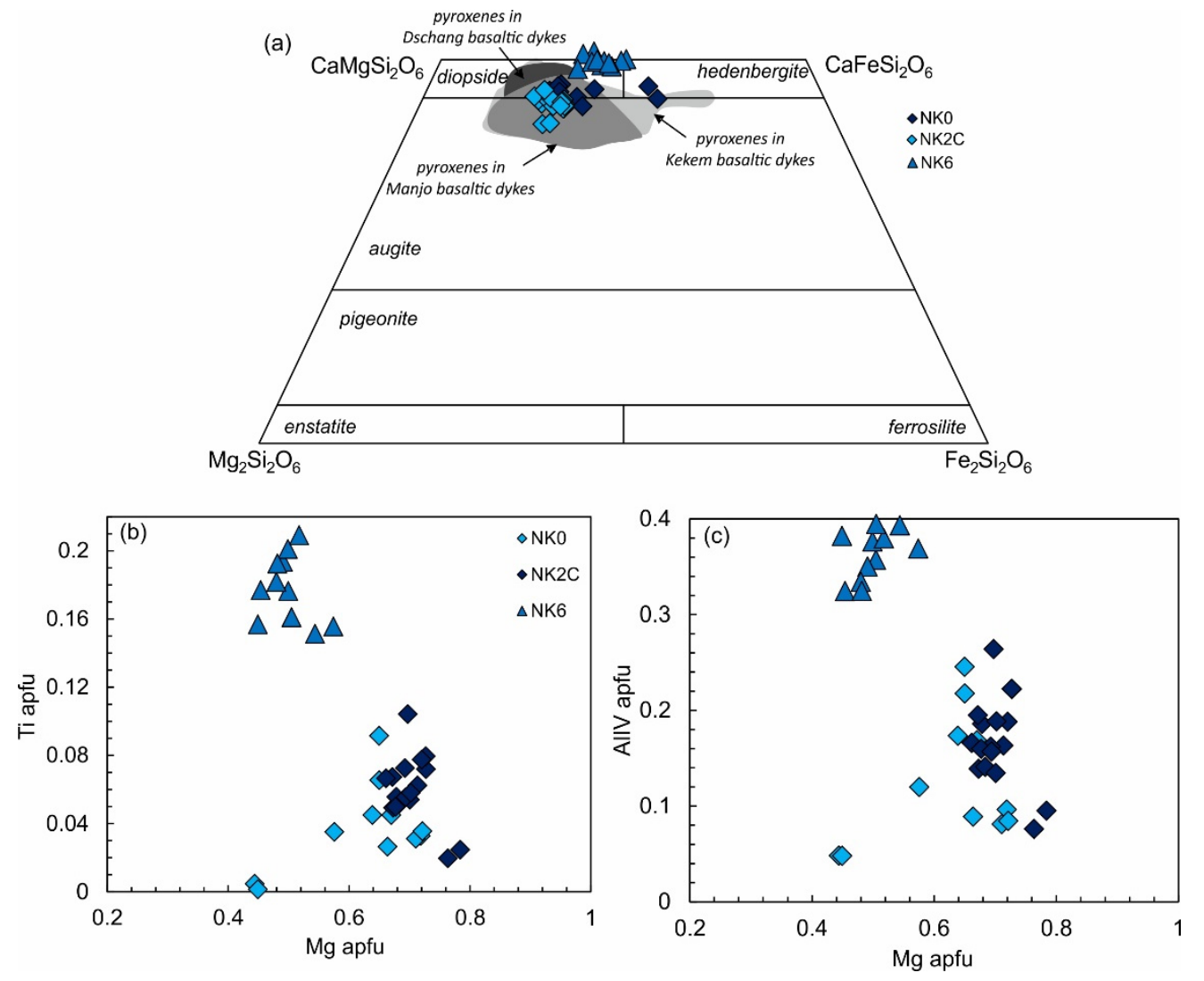

Figure 5. (a) Pyroxene compositions projected in the Ca-Mg-Fe diagram for the Njimom dykes. The data of clinopyroxenes of basaltic dykes from the Kekem, Dschang, and Manjo [5-7] are shown for comparison. (b) Ti vs. $\mathrm{Mg}$ (in apfu; atoms per formula unit) for Njimom clinopyroxenes. (c) $\mathrm{Al}^{\mathrm{IV}} \mathrm{vs}$. $\mathrm{Mg}$ (in apfu) for Njimom clinopyroxenes.

\subsubsection{Feldspars}

Plagioclase in the dyke NK6 is labradorite $\left(\mathrm{An}_{53-62}\right)$ and is accompanied by groundmass anorthoclase and minor sodic sanidine (Table 2; Figure 6). In the dykes NK2A and $\mathrm{NK} 2 \mathrm{C}$, plagioclase ranges from $\mathrm{An}_{62}$ to $\mathrm{An}_{33}$ (Figure 6). Anorthoclase and sodic sanidine have also been found in the groundmass. 


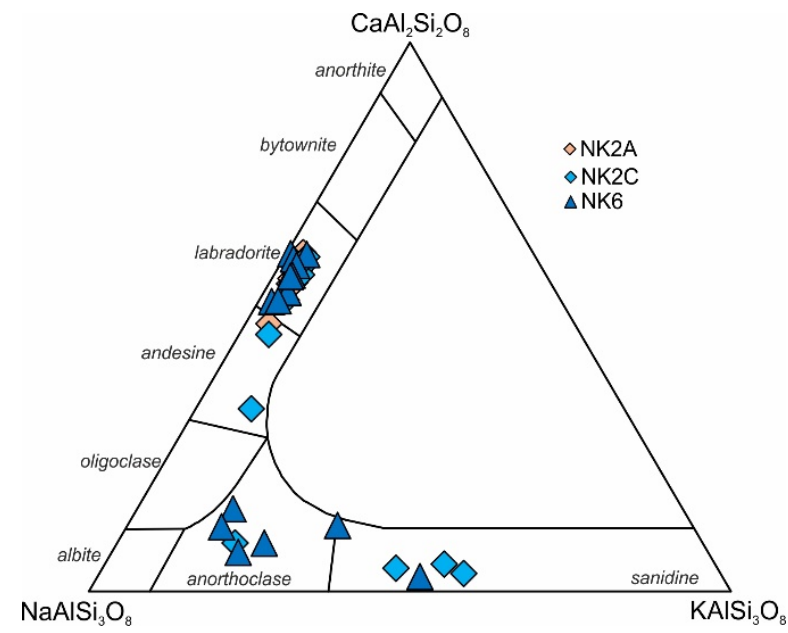

Figure 6. Feldspar compositions observed in the Njimom dykes.

\subsubsection{Oxides}

Cr-rich spinel has been found as inclusions in altered olivine in the dyke NK6 (Figure 7). The $\mathrm{Cr} \#(100 \times \mathrm{Cr} /(\mathrm{Cr}+\mathrm{Al})$ in atoms $)$ and $\mathrm{Mg} \#\left(100 \times \mathrm{Mg} /\left(\mathrm{Mg}+\mathrm{Fe}^{2+}\right.\right.$ in atoms $)$ range from 42 to 55 from 14 to 51 , respectively (Table 3 ). These spinels clearly have chemical composition different from spinels of Kekem dykes [5]. Titaniferous magnetite and ilmenite are the main oxides found in the Njimom dykes. Titaniferous magnetite in the dyke NK6 and NK2C has $62-84 \mathrm{~mol} \%$ ulvöspinel (Table 3). Ilmenite is compositionally less variable than titaniferous magnetite (ilm $94-99)$.

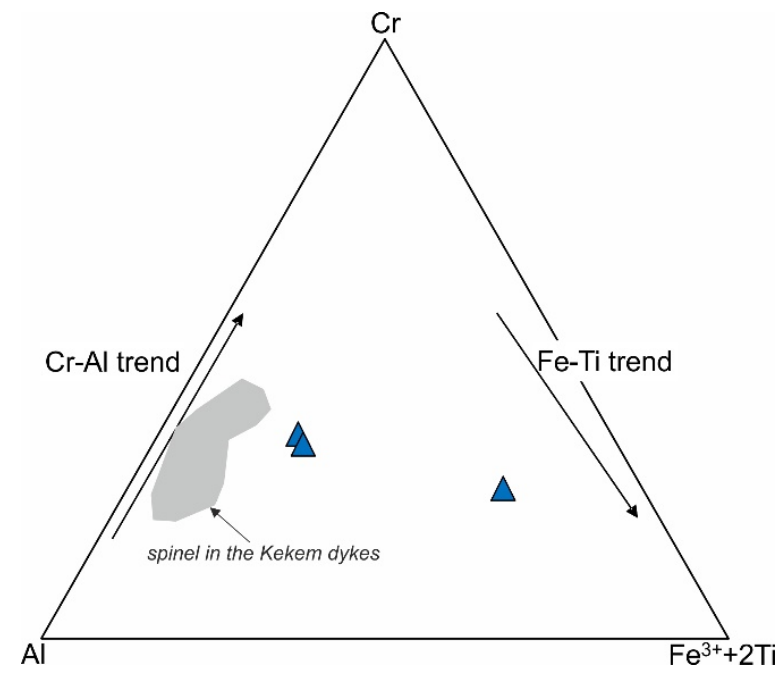

Figure 7. Chemical variations of chromium-rich spinels in the Njimom dykes. The field of spinels of the Kekem dykes is also shown [5].

\subsection{Whole-Rock Geochemistry}

The Njimom dykes described above range from alkali basalt through hawaiite to mugearite on the total alkali vs silica diagram from [18] (Figure 8). The variability in major element chemistry of the Njimom dykes could be the result of secondary or deuteric alteration of the rocks. Some care must be taken in interpreting the chemical data, for alteration has variably but visibly affected all the samples. Like hand specimen and petrographic observations indicate that some Njimom samples are altered indicating that their compositions may have been modified by late- or post-crystallization fluid-rock interactions. Alteration is reflected in a very general way in LOI (loss on ignition) values, which range widely from 3.34 to $9.21 \mathrm{wt}$ \% in the mafic dykes. Correlations between Ca and 
more alteration-resistant elements such as Ti or Zr are poor, suggesting that Ca contents have been affected significantly in some dykes. However, the alteration typically has little effect on the immobile trace elements (e.g., $[19,20])$. For these reasons, in this study we use only the alteration-resistant elements ( $\mathrm{Ti}, \mathrm{Zr}, \mathrm{Nb}, \mathrm{Y}, \mathrm{Th}$, and $\mathrm{REE}$ ) and their ratios for geochemical interpretations. To classify the mafic volcanic rocks, we use the incompatible trace-element classification scheme for altered volcanic rocks (Figure 9: [21]), which indicates that Njimom samples are alkaline basalts and hawaiites. Major and trace element contents of dykes from the Njimom area are listed in Table 4. The major element analyses are recalculated to $100 \mathrm{wt}$ \% LOI-free. The dykes that cross-cut the Precambrian granites have $\mathrm{SiO}_{2}$ contents between 46.0 and $47.5 \mathrm{wt} . \%, \mathrm{TiO}_{2}$ between 2.3 and $2.5 \mathrm{wt} . \%$ and moderate compatible trace element concentrations (e.g., $\mathrm{Cr}=260-280 \mathrm{ppm} ; \mathrm{Ni}=170-230 \mathrm{ppm}$ ). $\mathrm{MgO}$ varies from 5.9 to 9.2 wt. $\%$ and $\mathrm{Mg} \#\left(\mathrm{Mg} \#=\mathrm{Mg} \times 100 /\left(\mathrm{Mg}+\mathrm{Fe}^{2+}\right)\right)$ from 53 to 60 indicating that the samples moderately evolved. In contrast, the dykes that cross-cut the Precambrian mylonitic gneisses are more evolved. They have higher $\mathrm{SiO}_{2}$ contents (49.1-54.8 wt.\%) and lower $\mathrm{TiO}_{2}$ (1.7-2.0 wt.\%), $\mathrm{MgO}$ (4.4-7.1 wt.\%; Mg\# = 45-58), and compatible trace element concentrations (e.g., $\mathrm{Cr}=70-180$ ppm; $\mathrm{Ni}=30-110$ ppm). Major and trace element variations, using $\mathrm{Zr}$ as a differentiation index, are shown in Figure 10. The data for the two groups of dykes identified in the Njimom area plot in distinct fields in most major and trace element variation diagrams, particularly in the $\mathrm{SiO}_{2}, \mathrm{TiO}_{2}, \mathrm{Nb}$, $\mathrm{Y}$, and REE (Figure 10). The dykes that cross-cut the Precambrian granites (Figure 11a) are moderately light lanthanide element (LREE) enriched $\left(\mathrm{La}_{\mathrm{n}}=58\right.$ to 70 and $\mathrm{La}_{\mathrm{n}} / \mathrm{Yb}_{\mathrm{n}}$ from 7.6 to 8.7 , the subscript " $\mathrm{n}$ " means chondrite normalized). The dykes that intrude the Precambrian mylonitic gneisses are variably LREE enriched $\left(\mathrm{La}_{n} / \mathrm{Yb}_{\mathrm{n}}=7.2-10.6\right)$ and show small negative anomalies in $\mathrm{Eu}\left(\mathrm{Eu}_{\mathrm{n}} / \mathrm{Eu}^{*}=0.67-0.92\right.$, where $\mathrm{Eu}_{\mathrm{n}}$ is chondrite normalized europium and $\mathrm{Eu}^{*}=\left(\mathrm{Sm}_{\mathrm{n}} \times \mathrm{Gd}_{\mathrm{n}}\right)^{0.5}$; Figure $\left.11 \mathrm{~b}\right)$. Primitive mantle-normalized multielement patterns for dykes that intrude the granites have marked peaks in $\mathrm{Sr}$ are and smoothly decreasing normalized abundances from $\mathrm{Nb}$ to $\mathrm{Lu}$ (Figure 11c). Primitive mantle-normalized incompatible element patterns for dykes that intrude the mylonites show marked troughs at $\mathrm{Th}$ and $\mathrm{Ti}$ and weak troughs at $\mathrm{Nb}$ and $\mathrm{Sr}$ (Figure 11d).

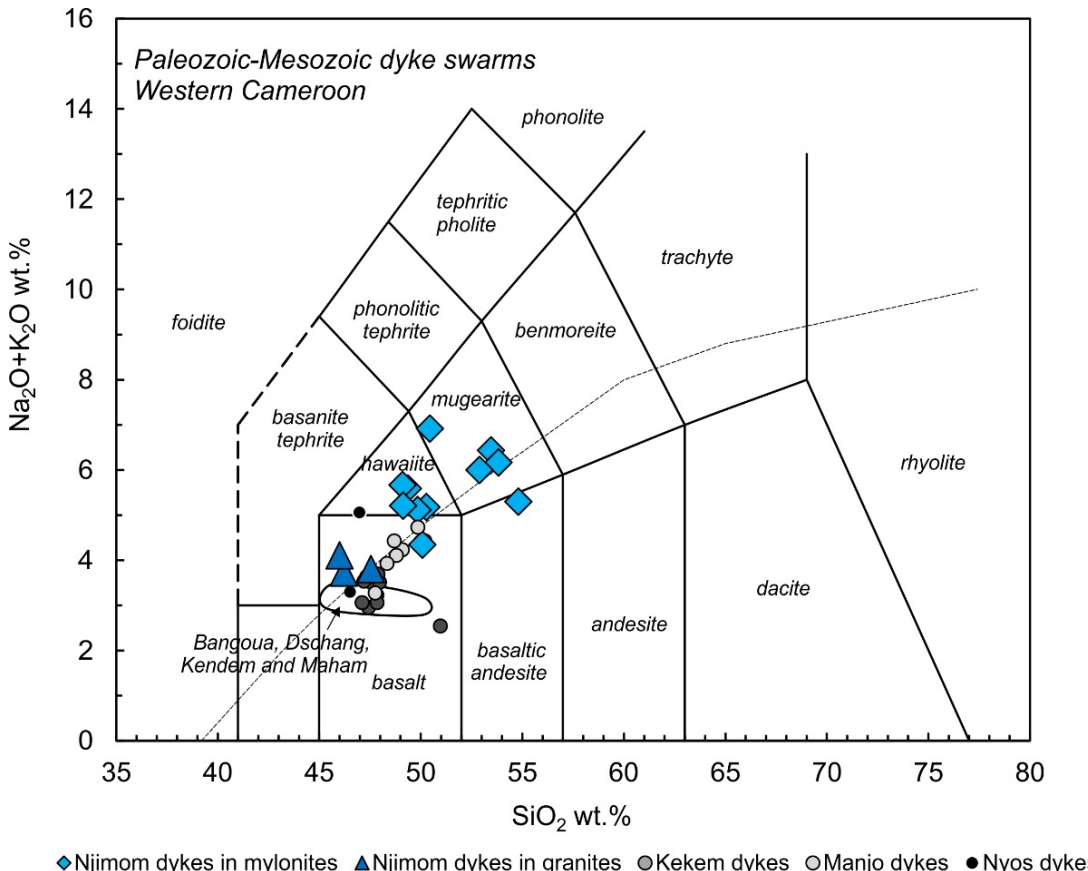

Figure 8. TAS classification diagram [18] for the Njimom dykes. The subalkaline-alkaline line is from [22]. Data of mafic dykes (Bangoua, Dschang, Maham, and Kendem) are from [7], Kekem dykes from [5], Manjo dykes from [6], Nyos dykes from [23]. 


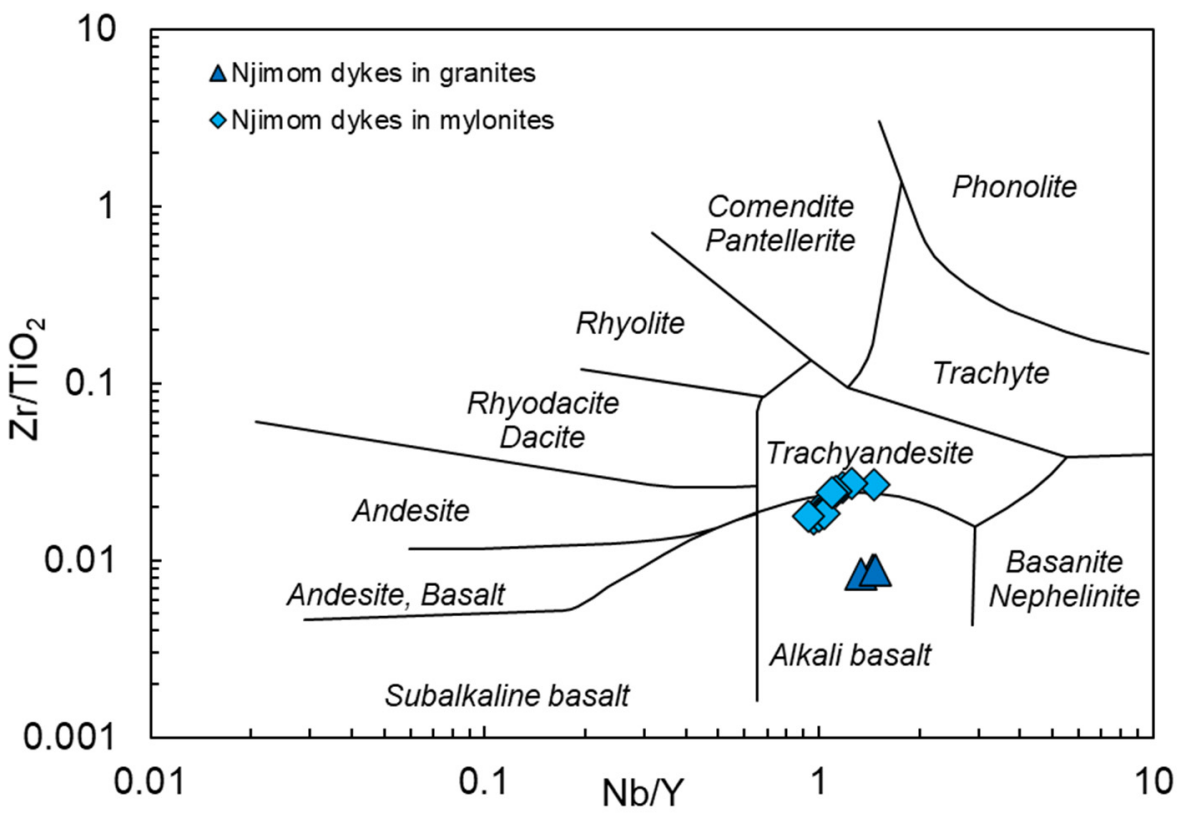

Figure 9. $\mathrm{Zr} / \mathrm{TiO}_{2}$ vs $\mathrm{Nb} / \mathrm{Y}$ discrimination diagram [21] showing the compositions of the Njimom dykes.
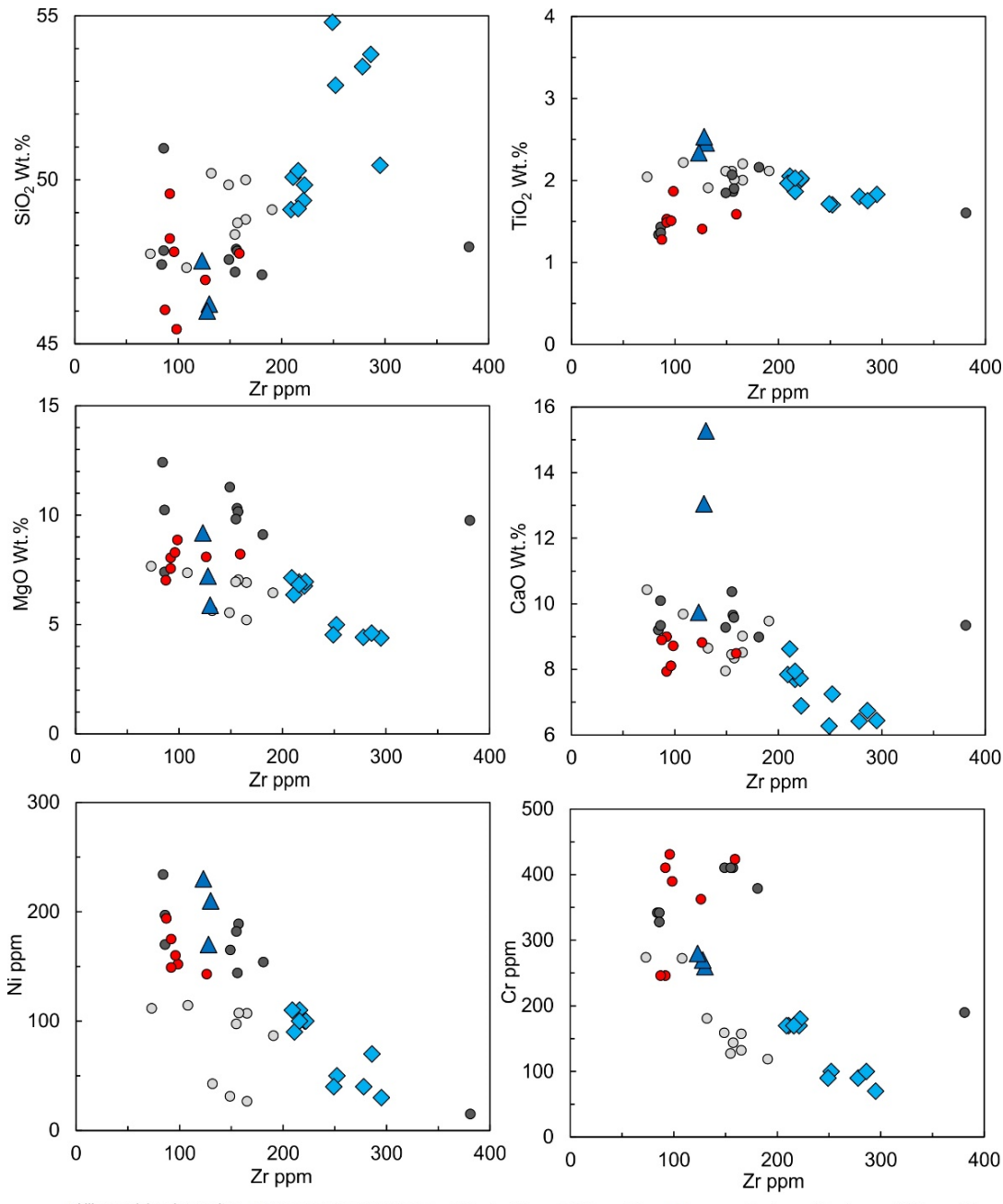

Figure 10. Cont. 

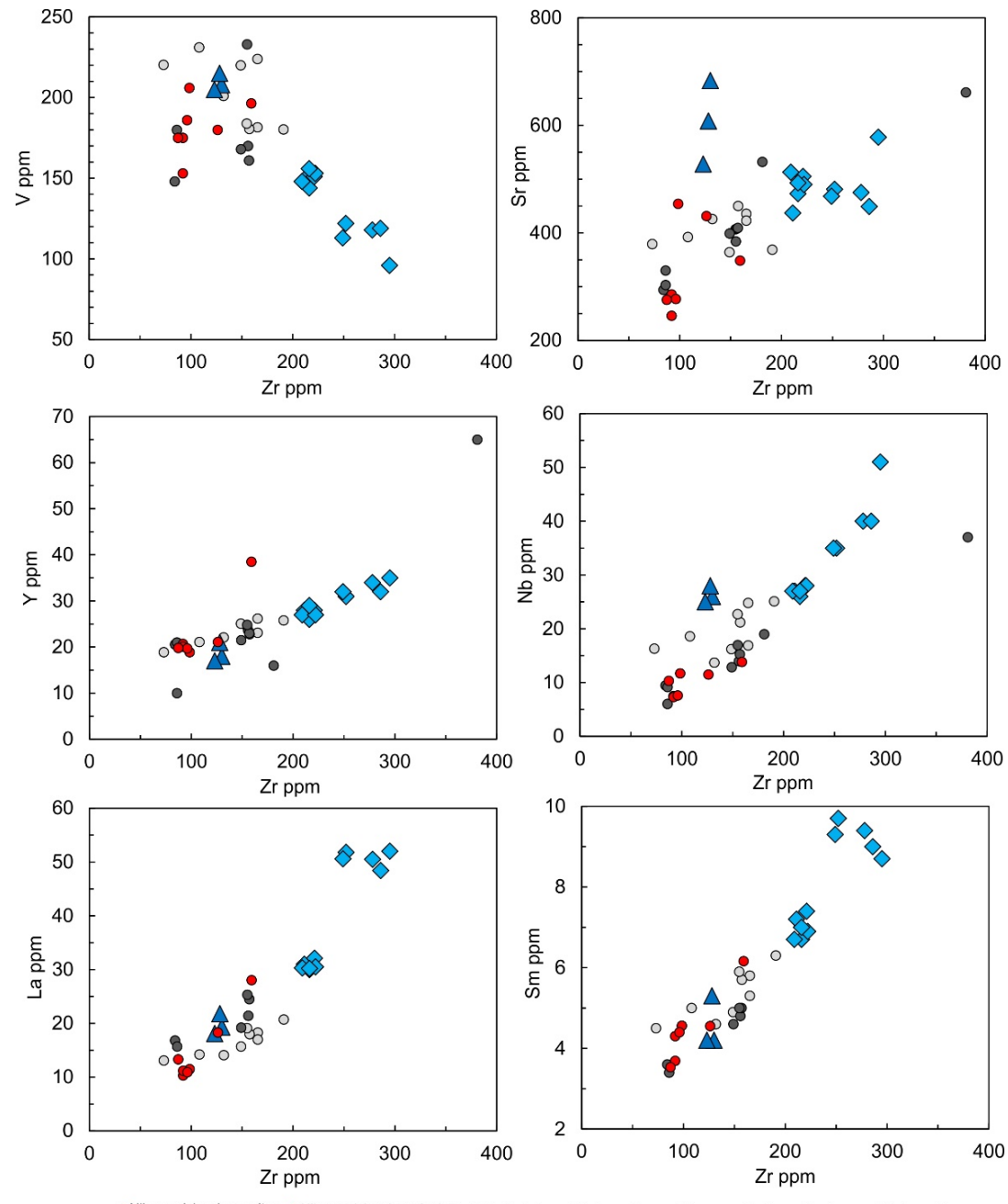

Figure 10. Major and trace element relationships among Njimom dykes. The data for the mafic dyke swarms (Bangoua, Maham, Dschang, Kendem, Manjo, and Kekem) are also shown for comparison [5-7].
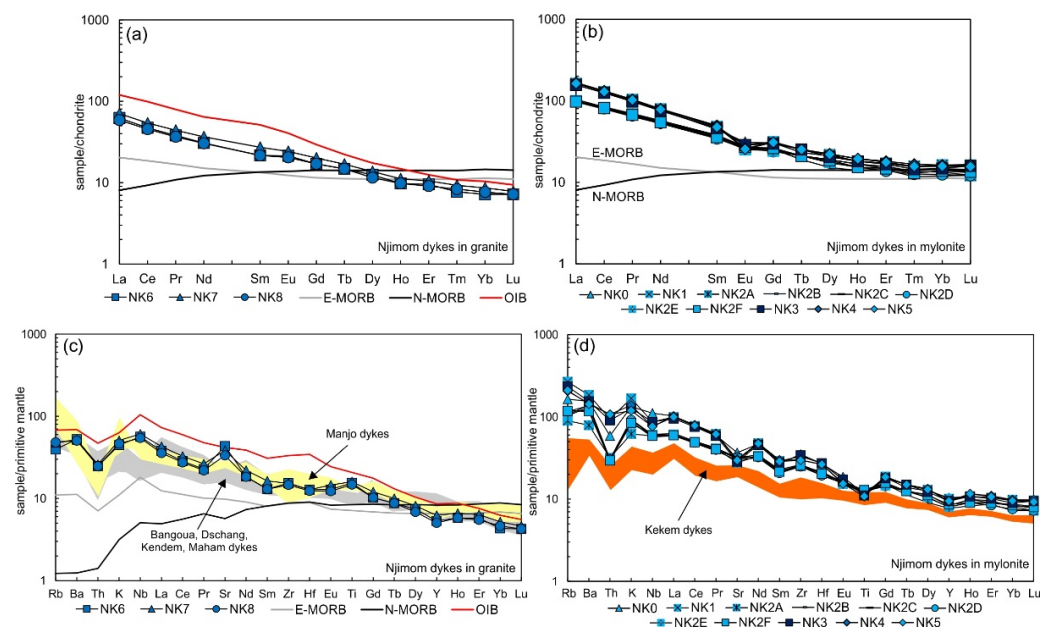

Figure 11. $(\mathbf{a}, \mathbf{b})$ Chondrite-normalized REE diagrams for Njimom dykes. The chondrite values used for normalization are those of [24]. Promethium is interpolated. N-MORB and E-MORB and OIB patterns are plotted with the values of [25]. (c,d) Primitive mantle-normalized incompatible element diagrams for Njimom dykes. Primitive mantle values are from [26]. Data from Manjo [6]; Kekem [5]; Bangoua, Maham, Dschang, and Kendem [7] are shown for comparison. 


\section{Discussion}

\subsection{Crustal Contamination}

The parental melts of the Njimom dykes must have experienced some differentiation before intruding the upper crust, as reflected in the evolved compositions of all the samples of this study. The Njimom dykes were derived by fractional crystallization of olivine, clinopyroxene, and plagioclase from Mg-rich basaltic melts, consistent with the petrographic assemblages observed in these dykes and their low magnesium numbers (45-60). Trace element ratios such as $\mathrm{Nb} / \mathrm{U}, \mathrm{Th} / \mathrm{Nb}$, and $\mathrm{La} / \mathrm{Nb}$ have been used to assess the role of crustal contamination in Njimom dykes because they are not strongly modified from their source material by partial melting or fractional crystallization processes. The continental crust has low $\mathrm{Nb} / \mathrm{U}(4.4-25)$ and high $\mathrm{La} / \mathrm{Nb}(1.6-2.6)$ and $\mathrm{Th} / \mathrm{Nb}(0.24-0.88)$ ratios (e.g., [27]). Ocean island basalt (OIB) and mid-ocean ridge basalt (e.g., E-MORB and $\mathrm{N}-\mathrm{MORB})$ are both characterized by high $\mathrm{Nb} / \mathrm{U}(>45)$ and low $\mathrm{La} / \mathrm{Nb}(0.8-1.1)$ and $\mathrm{Th} / \mathrm{Nb}(<0.1)$ ratios (e.g., $[25,28])$. In Njimom area, four dykes that intrude mylonites have $\mathrm{Nb} / \mathrm{U}$ (19.4-26.7), $\mathrm{Th} / \mathrm{Nb}(0.19-0.26)$, and $\mathrm{La} / \mathrm{Nb}$ (1.2-1.5) ratios suggesting small crustal input. Furthermore, these dykes show small negative $\mathrm{Nb}$ anomalies on the primitive mantle-normalized multi-element patterns (Figure 11d), reflecting small crustal contamination. In contrast, the dykes that cross-cut the granites show higher $\mathrm{Nb} / \mathrm{U}(43.3-50.0)$ and lower $\mathrm{Th} / \mathrm{Nb}(\sim 0.08)$ and $\mathrm{La} / \mathrm{Nb}(0.7-0.8)$ (Figure 12). These values are close to those of MORB and OIB suggesting no crustal contamination. Interestingly, the same observations can be made for other mafic dykes coming from the southern continental part of the CVL [5-7], thus suggesting that these mafic dykes remained preserved from crustal influence during their evolution.

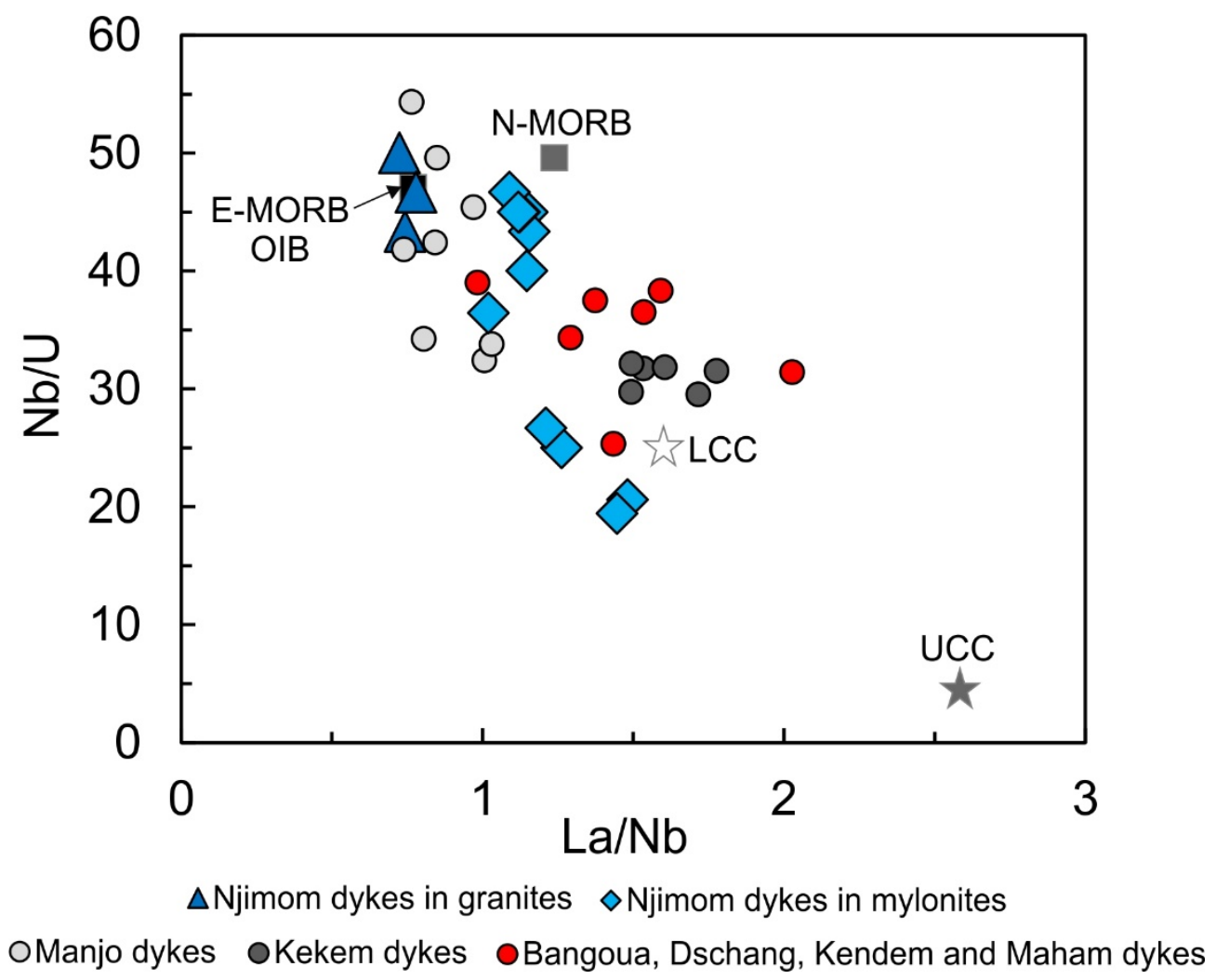

Figure 12. $\mathrm{La} / \mathrm{Nb}$ vs $\mathrm{Nb} / \mathrm{U}$ for the Njimom dykes. Data for upper continental crust (UCC) and lower continental (LCC) (star symbols) are from [27]. Data for oceanic basalts (OIB) and MORBs are from [25,28]. Data from Manjo [6]; Kekem [5]; Bangoua, Maham, Dschang, and Kendem [7] are shown for comparison. 


\subsection{Petrogenesis}

Two different dykes groups are present in the Njimom area. There are sufficient geochemical and mineralogical evidences to deduce that the dykes that cross-cut the granites and mylonites are not comagmatic. For example, the different $\mathrm{TiO}_{2}$ and $\mathrm{Al}_{2} \mathrm{O}_{3}$ contents in the clinopyroxenes of the Njimom dykes, as well as their different incompatible element patterns (Figure 11) cannot be produced by closed-system crystal fractionation of olivine, plagioclase, clinopyroxene, and opaque oxides.

Because none of the compositions observed in Njimom area can be considered as a mantle-derived primary magma, it is likely that multiple saturation of $\mathrm{Cr}$-spinel, olivine, clinopyroxene, and plagioclase occurred before the emplacement of all the magmas to shallow crustal levels. Therefore, we have modelled the extent of partial melting utilizing ratios of elements that are not modified by low to moderate fractionation of the phases mentioned in the preceding. In the $\mathrm{Nd} / \mathrm{Sm}$ versus $\mathrm{Tb} / \mathrm{Yb}$ diagram (Figure 13), the Njimom appear to have formed by small-degree melts $(2-5 \%)$ of sources slightly enriched in incompatible elements in the spinel stability field. The melts from garnet-bearing sources could be present only in very minor amounts. The mildly enriched geochemical characteristics of the Njimom dykes are evident from ratios of $\mathrm{Zr} / \mathrm{Nb}$ (5-8), $\mathrm{Zr} / \mathrm{Y}$ (6-9), $\mathrm{Nb} / \mathrm{Yb}$ (9-17), and Ti/V (68-114) that are similar to the values of (OIB) and E-MORB worldwide (e.g., [25]). Njimom dykes plot in the OIB field in the Ti-V diagram (Figure 14). The chemical composition of the Nijmom dykes is generally similar to that observed in Kendem, Dschang, Bangangte, and Manjo dyke swarms, particularly in the abundance of relatively immobile incompatible elements. These similarities warrant that broadly similar mantle sources, possibly melted in different degrees, and petrogenetic histories were involved in the petrogenesis of the Paleozoic-Mesozoic Cameroon dykes. From the genetic point of view, the geochemical characteristics of the Paleozoic-Mesozoic Cameroon dykes indicate that they cannot be considered as typical of plume-derived melts. We therefore argue that the genesis of these dykes could be referred to as passive rifting and melting of the shallow lithosphere, possibly followed by crustal contamination of the mafic magmas during the ascent through the Precambrian crust. This intracontinental extensional setting could have been caused by the opening of the South Atlantic Ocean.

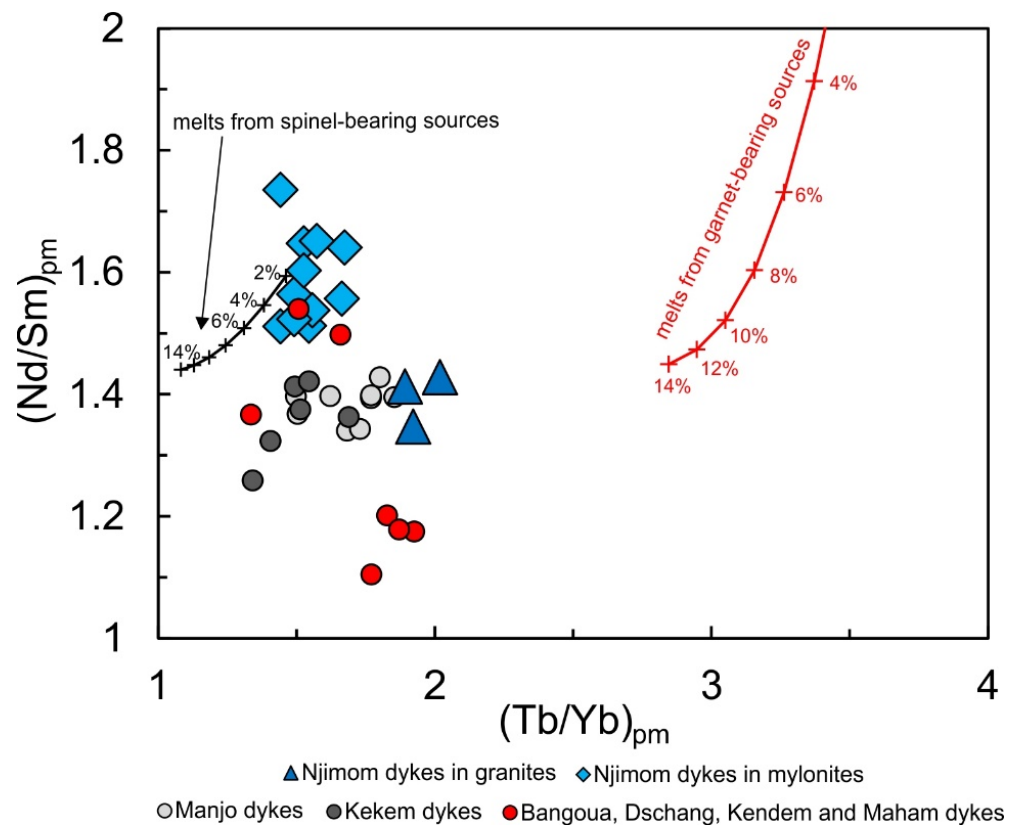

Figure 13. (Nd/Sm)pm vs. ( $\mathrm{Tb} / \mathrm{Yb})$ pm diagram illustrating non-modal fractional melting models for the Njimom dykes. The values along curves are the degrees of partial melting. The composition of the source is the lithospheric mantle of [29]. Mode of the source, eutectics, and partition coefficients after [30]. 


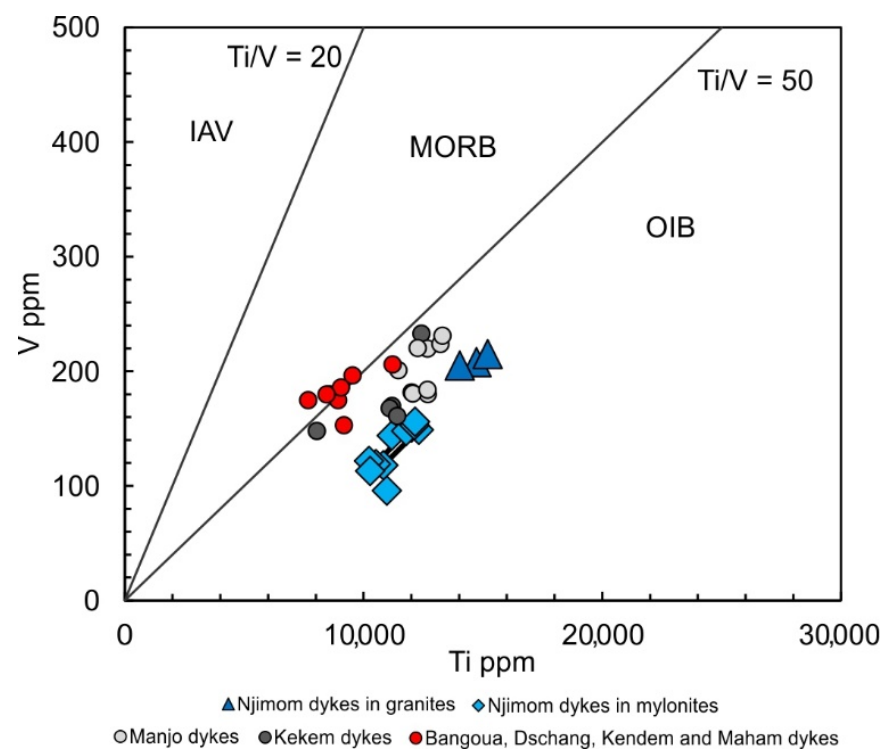

Figure 14. Ti-V diagram (after [31]) for Njimom dykes.

\subsection{Geodynamic Implications}

The Njimom mafic dykes have a strong NW-SE preferred orientation. In the Riedel fracture model for lineaments of Cameroon [8,9], it corresponds to the direction of major $\sigma 1$ regional constraints. Previous studies on basaltic dykes in the southern continental part of the CVL record measurements of ca. 30 dykes (e.g., $[9,32,33]$ ). Two dykes at Kendem (near Mamfe) show a similar orientation. The Ar/Ar age of 192.10 $\pm 7.45 \mathrm{Ma}$ recorded in Kendem (the lonely existing age for basaltic dykes of the southern continental part of the CVL) is older compared to the age of 125 million years considered as the beginning of the opening of the South Atlantic Ocean. Ar/Ar age significantly older (421.3 $\pm 3.5 \mathrm{Ma})$ has been obtained for a basaltic dyke outcropping along the "Dschang's cliff' road at ca. $10 \mathrm{~km}$ to the west of the city of Dschang [7]. On a regional basis, the NW-SE direction is also known as the Benue direction in Cameroon which is linked to the Benue Through aulacogen initiated during the opening of the southern Atlantic (e.g., [34]). Alkaline magmatism and abundance of crustal xenoliths in some studied dykes can thus be linked to the paroxysmal stage preceding the opening of the Atlantic Ocean while transitional/tholeiitic affinities recorded for older (Paleozoic) basaltic dykes may better indicate simple reactivations of Precambrian fractures. The Njimom dykes could represent the transition between an older tholeiitic magmatism and the dominant Cretaceous alkaline magmatism of the Cameroon Volcanic Line.

\section{Conclusions}

On the basis of field, mineralogical and whole-rock geochemical data of the Njimom dykes, we present the following conclusions:

(1) Two groups of mafic dykes that crosscut the Neoproterozoic basement rocks have been observed. A first group intrudes the mylonites whereas the second group intrudes the granites;

(2) The Njimom dykes are alkaline basalts and hawaiites with a weakly porphyritic to aphyric texture and contain clinopyroxene, plagioclase, \pm altered olivine and opaque oxides;

(3) Geochemical variations at each dyke group are compatible with fractional crystallization with no or little crustal contamination, whereas the two dyke groups represent distinctive magma sources in the mantle;

(4) Geochemical characteristics of the Njimom dykes can be modelled by partial melting $(2-5 \%)$ of lherzolite slightly enriched in incompatible elements in the spinel stability field; 
(5) The geochemical features of Njimom dykes are similar to those observed in the Paleozoic and Mesozoic dykes recorded in the southern continental part of the Cameroon Volcanic Line, suggesting a similar mantle source evolution;

(6) The magmatic activity in the Njimom area probably was synchronous with the initial phase of the opening of the southern Atlantic Ocean.

\begin{abstract}
Author Contributions: Conceptualization, N.-A.K.K., N.A.W.S., B.K.N., A.A.N., J.D.S., D.T.T., J.P.T., and C.C.; methodology, C.C. and J.P.T.; formal analysis, C.C. and J.P.T.; investigation, N.-A.K.K., N.A.W.S., B.K.N., A.A.N., J.D.S., D.T.T., J.P.T., and C.C.; resources, C.C. and J.P.T.; writing-original draft preparation; writing-review and editing, C.C. and J.P.T.; visualization, C.C. and J.P.T.; supervision, C.C. and J.P.T.; funding acquisition, C.C. All authors have read and agreed to the published version of the manuscript.
\end{abstract}

Funding: Funds for mineral and whole-rock analyses were provided by MIUR 2017 (grant 20178LPCPW_004 to C. Cucciniello).

Data Availability Statement: The whole-rock geochemical (major and trace element) and mineralogical data used in this manuscript are original and reported in the tables.

Acknowledgments: C. Cucciniello thanks Roberto de' Gennaro for assistance in the SEM-EDS laboratory. The manuscript was improved by the journal reviews of two anonymous referees and comments from the editor Jesus Martinez-Frias. Constant support provided by Managing Editor Lionel Zheng during the manuscript production stage is appreciated.

Conflicts of Interest: The authors declare no conflict of interest. The funders had no role in the design of the study; in the collection, analyses, or interpretation of data; in the writing of the manuscript, or in the decision to publish the results.

\title{
References
}

1. Déruelle, B.; Ngounouno, I.; Demaiffe, D. The 'Cameroon Hot Line' (CHL); a unique example of active alkaline intraplate structure in in both oceanic and continental lithospheres. Comp. Rend. Geosci. 2007, 339, 589-600. [CrossRef]

2. Rankenburg, K.; Lassiter, J.C.; Brey, G. The role of continental crust and lithospheric mantle in the genesis of Cameroon volcanic line lavas: Constraints from isotopic variations in lavas and megacrysts from the Biu and Jos Plateaux. J. Petrol. 2005, 46, 169-190. [CrossRef]

3. Mbowou, G.I.B.; Lagmet, C.; Nomade, S.; Ngounouno, I.; Déruelle, B.; Ohnenstetter, B. Petrology of the Late Cretaceous peralkaline rhyolites (pantellerite and commendite) from Lake Chad, Central Africa. J. Geosci. 2012, 57, 127-141. [CrossRef]

4. Tchikankou, N.L.N.; Kamgang, P.; Chazot, G.; Agranier, A.; Bellon, H.; Nonnotte, P.; Tchuimegnie Ngongang, N.B.; Kwekam, M. Mantle source evolution beneath the Cameroon Volcanic Line: Geochemical and geochronological evidences from Fotouni volcanic series, Western Cameroon. Arab. J. Geosci. 2020, 13, 1-20. [CrossRef]

5. Tchaptchet, T.D.; Wambo Simeni, N.A.; Keutchafo Kouamo, N.A.; Tchouankoue, J.P.; Cucciniello, C. Geology, mineralogy and geochemistry of the Kekem dyke swarm (Western Cameroon): Insights into Paleozoic-Mesozoic magmatism and geodynamic implications. Comp. Rend. Geosci. 2017, 349, 175-185. [CrossRef]

6. Keutchafo Kouamo, N.A.; Tchatptchet, T.D.; Ngueguim Tezanou, A.L.; Simeni Wambo, N.A.; Tchouankoue, J.P.; Cucciniello, C. Petrogenesis of basaltic dikes from the Manjo area (Western Cameroon): Insights into the Paleozoic magmatism at the northern margin of the Congo craton in Cameroon. Arab. J. Geosci. 2019, 12, 281. [CrossRef]

7. Tchouankoue, J.P.; Simeni Wambo, N.A.; Kagou Dongmo, A.; Li, X.H. ${ }^{40} \mathrm{Ar} /{ }^{39} \mathrm{Ar}$ dating of basaltic dyke swarm in Western Cameroon: Evidence of Late Paleozoic and Mesozoic magmatism in the corridor of the Cameroon Line. J. Afr. Earth Sci. 2014, 93, 14-22. [CrossRef]

8. Moreau, C.; Regnoult, J.-M.; Déruelle, B.; Robineau, B. A new tectonic model for the Cameroon line; Central Africa. Tectonophysics 1987, 139, 317-334. [CrossRef]

9. Simeni Wambo, N.A.; Tchaptchet Tchato, D.; Ngo Belnoun, R.N.; Tchouankoue, J.P.; Ganwa, A.A. Structural Relationship between Brittle Deformation and Paleozoic to Mesozoic Basalt Dykes in the Precambrian Basement of the Southern Continental Part of the Cameroon Volcanic Line. Int. J. Geosci. 2017, 8, 318-331. [CrossRef]

10. Djouka-Fonkwé, M.L.; Schulz, B.; Schüssler, U.; Tchouankoué, J.P.; Nzolang, C. Geochemistry of the Bafoussam Pan-African Iand S-type granitoids in western Cameroon. J. Afr. Earth Sci. 2008, 50, 148-167. [CrossRef]

11. Tchouankoue, J.P.; Li, X.-H.; Ngo Belnoun, R.N.; Mouafo, L.; Ferreira, V.P. Timing and tectonic implications of the Pan-African Bangangte syeno-monzonite; West Cameroon: Constraints from in-situ zircon U-Pb age and Hf-O isotopes. J. Afr. Earth Sci. 2016, 124, 94-103. [CrossRef]

12. Ntiéche, B.; Moham, M.; Moundi, A. Granitoids of the Magba Shear Zone West Cameroon; Central Africa: Evidence for Emplacement under Transpressive Tectonic regime. J. Geol. Soc. India 2017, 89, 33-46. [CrossRef] 
13. Njonfang, E.; Ngako, V.; Kwekam, M.; Affaton, P. Calc-alkaline orthogneisses of the Foumban-Bankim shear zone: Witnesses of an internal zone of a Pan-African active margin. Comp. Rend. Geosci. 2006, 338, 606-616. [CrossRef]

14. Njonfang, E.; Ngako, V.; Moreau, C.; Affaton, P.; Diot, H. Restraining bends in high temperature shear zone: «the Central Cameroon Shear Zone»; Central Africa. J. Afr. Earth Sci. 2008, 52, 9-20. [CrossRef]

15. Kamguia Kamani, M.S.; Wang, W.; Tchouankoue, J.-P.; Huanga, S.F.; Yomeun, B.; Xue, E.K.; Gui-Mei Lu, G.-M. Neoproterozoic syn-collision magmatism in the Nkondjock region at the northern border of the Congo craton in Cameroon: Geodynamic implications for the Central African orogenic belt. Precambrian Res. 2021, 353, 106015. [CrossRef]

16. Moundi, A.; Menard, J.-J.; Reusser, E.; Tchoua, F.M.; Dietrich, V.J. Découverte de basaltes transitionnels dans le secteur continentalde la ligne du Cameroun (massif du Mbam; Ouest-Cameroun). C. R. Acad. Sci. 1996, 322, 831-837.

17. Ngako, V.; Affaton, P.; Njonfang, E. Pan-African tectonics in northern Cameroon: Implication for the history of western Gondwana. Gondwana Res. 2008, 14, 509-522. [CrossRef]

18. Le Bas, M.J.; Le Maitre, R.W.; Streckeisen, A.L.; Zanettin, B.A. chemical classification of volcanic rocks based on the total alkali-silica diagram. J. Petrol. 1986, 27, 745-750. [CrossRef]

19. Cucciniello, C.; Choudhary, A.K.; Pande, K.; Sheth, H. Mineralogy, geochemistry and ${ }^{40} \mathrm{Ar} /{ }^{39} \mathrm{Ar}$ geochronology of the Barda and Alech complexes, Saurashtra, northwestern Deccan Traps: Early silicic magmas derived by flood basalt fractionation. Geol. Mag. 2019, 156, 1668. [CrossRef]

20. Cucciniello, C.; Demonterova, E.I.; Sheth, H.; Pande, K.; Vijayan, A. ${ }^{40} \mathrm{Ar} /{ }^{39} \mathrm{Ar}$ geochronology and geochemistry of the Central Saurashtra mafic dyke swarm: Insights into magmatic evolution; magma transport; and dyke flow relationships in the northwestern Deccan Traps. Bull. Volcanol. 2015, 77, 45. [CrossRef]

21. Winchester, J.A.; Floyd, P.A. Geochemical discrimination of different magma series and their differentiation products using immobile elements. Chem. Geol. 1977, 20, 325-343. [CrossRef]

22. Irvine, T.N.; Baragar, W.R.A. A guide to the chemical classification of the common rocks. Can. J. Earth Sci. 1971, 8, 523-548. [CrossRef]

23. Aka, F.T.; Hasegawa, T.; Nche, L.A.; Asaah, A.N.E.; Mimba, M.E.; Teitchou, I.; Ngwa, C.; Miyabuchi, Y.; Kobayashi, T.; Kankeu, B.; et al. Upper Triassic basalt dykes of Lake Nyos; Cameroon (West Africa) I: K-Ar age evidence within the context of Cameroon Line magmatism; and the tectonic significance. J. Afr. Earth Sci. 2018, 141, 49-59. [CrossRef]

24. Boynton, W.B. Cosmochemistry of rare earth elements: Meteorite studies. In Rare Earth Element Geochemistry; Henderson, P., Ed.; Elsevier: Amsterdam, The Netherlands, 1984; pp. 63-114.

25. Sun, S.-S.; McDonough, W.F. Chemical and isotopic systematics of oceanic basalts: Implications for mantle composition and processes. Geol. Soc. Lond. Spéc. Publ. 1989, 42, 313-345. [CrossRef]

26. Lyubetskaya, T.; Korenaga, J. Chemical composition of Earth's primitive mantle and its variance: Method and results. J. Geophys. Res. 2007, 112. [CrossRef]

27. Rudnick, R.L.; Gao, S. Composition of the continental crust. In Treatise on Geochemistry 3 The Crust; Rudnick, R.L., Ed.; Elsevier: Amsterdam, The Netherlands, 2003; pp. 1-64.

28. Hofmann, A.W.; Jochum, K.P.; Seufert, M.; White, W.M. Nd and Pb in oceanic basalt: New constraints on mantle evolution. Earth Planet. Sci. Lett. 1986, 79, 33-45. [CrossRef]

29. McDonough, W.F. Constraints on the composition of the continental lithospheric mantle. Earth Planet. Sci. Lett. 1990, 101, 1-18. [CrossRef]

30. Melluso, L.; Cucciniello, C.; le Roex, A.P.; Morra, V. The geochemistry of primitive volcanic rocks of the Ankaratra volcanic complex; and source enrichment processes in the genesis of the Cenozoic magmatism in Madagascar. Geochim. Cosmochim. Acta 2016, 185, 435. [CrossRef]

31. Shervais, J.J. Ti-V plots and the petrogenesis of modern and ophiolitic lavas. Earth Planet. Sci. Lett. 1982, 59, 101-118. [CrossRef]

32. Tchouankoue, J.P.; Simeni Wambo, N.A.; Kagou Dongmo, A.; Wörner, G. Petrology; geochemistry; and geodynamic implications of basaltic dyke swarms from the southern continental part of the Cameroon Volcanic Line; Central Africa. Open Geol. J. 2012, 6, 72-84. [CrossRef]

33. Kouankap Nono, G.D.; Wotchoko, P.; Ganno, S.; Njinchuki, D.N.; Nzenti, J.P.; Suh, C.E. Petrochemical characterization of two distinct types of dolerites from Bafoussam area; West Cameroon. Intern. J. Geosci. 2013, 4, 1131-1144. [CrossRef]

34. Béa, A.; Cochemé, J.-J.; Trompette, R.; Affaton, P.; Soba, D.; Sougy, J. Grabens d'âge Paléozoïque inférieur et volcanisme tholéiitique associé dans la région de Garoua au Nord-Cameroun. J. Afr. Earth Sci. 1990, 10, 657-667. [CrossRef] 